\title{
Experimental investigation of the vorticity generation within a spilling water wave
}

\author{
By DANA DABIRI AND MORTEZA GHARIB \\ Graduate Aeronautics Laboratories, California Institute of Technology, \\ Pasadena, CA 91125, USA
}

(Received 13 July 1995 and in revised form 26 July 1996)

Sources of vorticity are examined for a laboratory-generated spilling breaking wave. Two cases are studied. For the first case, based on the breaker height, the Reynolds and Froude numbers are 7370 and 2.04, respectively. The breaker is preceded by $1 \mathrm{~mm}$ wavelength capillary waves, with the largest amplitude-to-wavelength ratio equal to 0.18 . For this case, it is found that the dominant source of vorticity flux is a viscous process, due to the deceleration of a thin layer of the surface fluid. For the second case, the Reynolds and Froude numbers based on the wave height are 1050 and 1.62, respectively. No breaking is observed for this case; rather a capillary-gravity wave is observed with $4 \mathrm{~mm}$ wavelength capillaries preceding the gravity wave. The largest amplitude-to-wavelength ratio of these capillaries is 0.28 . This case shows that capillary waves do not contribute to the vorticity flux; rather the only dominant source of the vorticity flux into the flow is the free-surface fluid deceleration.

Lastly, a thin free-surface jet that is relatively vorticity-free is found to precede the spilling breaker. Analyses suggest that our wave-breaking phenomena can be modelled by a hydraulic jump phenomenon where the Froude number is based on the thickness of the free-surface jet, and on the velocity of the free-surface jet just prior to breaking. We believe this to be a more physically descriptive value of the Froude number. For the high-speed case, the Froude number based on the thickness of the free-surface jet is 4.78 , while for the lower-speed case it is 2.14 .

\section{Introduction}

Breaking waves are a phenomenon common in nature. Historically, breakers have been categorized as either spilling breakers, where the water spills down the front face of the wave, or plunging breakers, where a jet emanating from the water crest falls into the base of the wave. The most commonly occurring breakers are spilling breaking waves, which occur mostly in deep ocean waters. These spilling breakers, known as white caps, are responsible for most of the air/sea interactions, and consequently for the air entrainment which is important for the life under the sea (Banner \& Peregrine 1993). Also, one can easily observe wave breaking phenomena in shallow-water hydraulic jumps or bow and stern waves of boats.

Several investigators have contributed to our basic understanding of spilling breaking waves. Elementary models describe breaking as a roller residing over the front face of a non-breaking wave. Later studies (Banner \& Phillips 1974; Peregrine \& Svendson 1978) have shown that the flow just below the breaker is not a roller, but a thin turbulent region beneath the spilling breaker. The turbulent layer extends downstream at the free surface, and decays with increasing distance from the breaker. Battjes \& Sakai (1981) used a hydrofoil to induce spilling breakers, and obtained 
velocity profiles at various stations downstream of the hydrofoil. They determined that the turbulent wake downstream of the separation at the surface is self-preserving, indicating the possible existence of a shear layer. Tulin \& Cointe (1986) and Cointe \& Tulin (1994) present a detailed and comprehensive theory of steady wave breaking. Their results were compared with experimental work performed by Duncan (1981, 1983) and found to agree quite well. In their most recent work, Duncan \& Philomin (1994) showed some of the features associated with the development of spilling breaking waves. Using the particle image velocimetry (PIV) technique, Lin \& Rockwell (1994) were able to map the flow field directly beneath the spilling breaker, and were therefore able to show the existence of a shear layer beneath the breaking wave. Through further study, they were also able to characterize the evolution of wave breaking (Lin \& Rockwell 1995). An interesting approach to studying ship-generated wave breaking is given by Miyata \& Inui (1984), where the authors suggest that the proper Froude number necessary to describe these breakers should be based on the ship draught rather than its length.

As these studies have shown, there is a connection between the breaking process and the near-surface turbulence and vorticity fields generated just below the breaker. The question that arises, then, is where does this vorticity come from? Banner \& Phillips (1974) suggest that 'incipient breaking in a steady wave train is characterized by the occurrence of stagnation points at wave crests, but not necessarily by discontinuities in slope'. Longuet-Higgins (1992) predicts that parasitic capillaries seen on the forward slope of gravity waves contribute significantly to the vorticity seen in the crest roller. After a series of analyses, he showed that due to surface curvature, there exists a Stokes layer $\delta=(2 v / \gamma)^{2}$, where $v$ is the kinematic viscosity and $\gamma$ is the radian frequency of the wave, within which vorticity of magnitude $\omega \approx 2(a \kappa)^{2} \gamma$ is generated, and beyond which vorticity escapes ( $a$ is the wave amplitude, $\kappa$ is the wavenumber, and $a \kappa$ describes the wave steepness). After detailed observations, Lin \& Rockwell (1994) conclude that the sharp surface curvature serves as a source of vorticity, thereby giving rise to a separated mixing layer. They also observe that the region between the free surface and the mixing layer contains vorticity levels which are small compared with the mixing layer itself. Lastly, Hornung, Willert \& Turner (1995) suggest that the substantial vorticity seen downstream of a hydraulic jump is due to the entrainment of bubbles during breaking. This was further explained by suggesting that the circulation around a loop, drawn directly beneath the free surface on one side and stretching into the fluid bulk on all other sides such that the loop incorporates a bubble, is non-zero. They also point out that the entrainment of vorticity is connected to how the circulation loop is drawn.

It is therefore the purpose of this paper to shed some light on the origins of the source of vorticity seen within a spilling breaking wave. Specifically, the questions that will be answered are (a) What is the source of the vorticity seen beneath and downstream of the breaker? (b) Do capillary waves contribute to the vorticity? (c) Do we need large breaking and therefore air entrainment in order to generate vorticity?

\section{Experimental set-up}

\subsection{The test facility}

In order to answer these questions, a series of spilling wave breaking experiments were performed in a closed-loop water tunnel facility at Caltech. The water tunnel has a contraction ratio of $4: 1$, with a $15.2 \times 15.2 \times 61 \mathrm{~cm}^{3}$ test section. Previous methods for generating spilling breaking waves have been to situate a hydrofoil some distance below the water level in a water tunnel. This causes the fluid above the hydrofoil to 


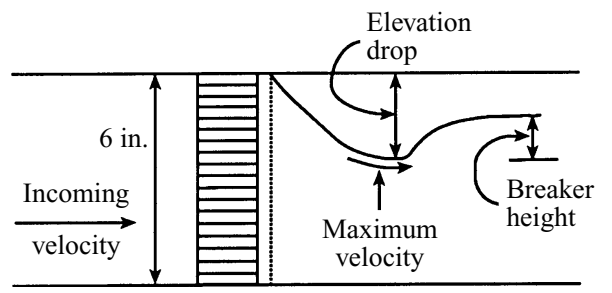

FIGURE 1. Wave breaking experimental set-up (not to scale).

accelerate, and therefore generates spilling breaking waves. For the present experiments, an original technique is devised to generate spilling breaking waves. A $15.2 \times 15.2 \times 2.54 \mathrm{~cm}^{3}$ honeycomb section with a screen is placed at the beginning of the test section, where the honeycomb straws are $2.3 \mathrm{~mm}$ in diameter, and the wire screen is $50 \mathrm{mesh} / \mathrm{inch}$ stainless steel (see figure 1). Owing to the pressure drop across the honeycomb/screen section, the fluid is accelerated to a maximum velocity, while dropping in height, and thereby spilling breaking waves are generated.

\subsection{The DPIV set-up and acquisition system}

Figure 2(a) shows a schematic for the experimental set-up for data acquisition. The flow is seeded with $14 \pm 5 \mu \mathrm{m}$ silver-coated hollow glass spheres with a specific gravity of $1.4 \mathrm{~g} \mathrm{~cm}^{-3}$. Through a series of optics and mirrors, a laser light sheet with a maximum thickness of $1 \mathrm{~mm}$ is generated. This light sheet is then set parallel to the flow direction allowing the wave to be viewed before, during, and after breaking. The digital particle image velocimetry (DPIV) system is used to record images onto a laser disk, which are subsequently digitized onto the hard disk. The DPIV system is based on the cross-correlation technique. Therefore, images are exposed only once and are recorded at 30 f.p.s. For analysis, a small interrogation window uniformly steps through the same locations within sequential pairs of images, performing a crosscorrelation analysis at each location. Finally, a Gaussian curve-fit is used to obtain sub-pixel accuracy of the cross-correlation peak. Once this is done at all locations within the image pairs, a displacement field in pixel values is obtained. By calibrating the pixels to spatial values, and knowing the time difference between exposures, the velocity field can be obtained. A more detailed description of the cross-correlation technique used here can be found in Willert \& Gharib (1991). Unfortunately, previous cross-correlating hardware limited the technique to slow flows, since each image could only be exposed at the same location within the frame, as shown in the second timing diagram of figure $2(b)$. Therefore, the pulse separation, $\Delta t$, was always limited to $1 / 30 \mathrm{~s}$, limiting this approach to maximum velocities of $\sim 10 \mathrm{~cm} \mathrm{~s}^{-1}$ for typical applications. However, the present modified system used for the present experiments is capable of exposing full-frame pulsed images at any point within the frame. Therefore, it is possible to expose the first image of the cross-correlating pairs very near its end, while exposing the second image of the cross-correlating pairs very near its beginning, as shown in figure $2(b)$ (iii). Consequently, this allows very short pulse separations between two image pairs, denoted by $\Delta t$ in figure $2(b)$ (iii), thereby making cross-correlation DPIV measurements possible at high speeds. For the present experiment, $\delta t_{1}=\delta t_{2}=150 \mu \mathrm{s}$, and $\Delta t=200 \mu \mathrm{s}$. The typical field of view is $2.5 \times 1.8 \mathrm{~cm}^{2}$. This area is digitized at $768 \times 480$ pixels $^{2}$, and processed with an interrogating window size of $32 \times 32$ pixels $^{2}$ with a step size of $16 \times 16$ pixels $^{2}(50 \%$ window overlap). Particles images are $28.8 \pm 0.5 \mu \mathrm{m}$. The uncertainties are $1.4 \%$ in the velocities and $3.9 \%$ in the vorticities. 


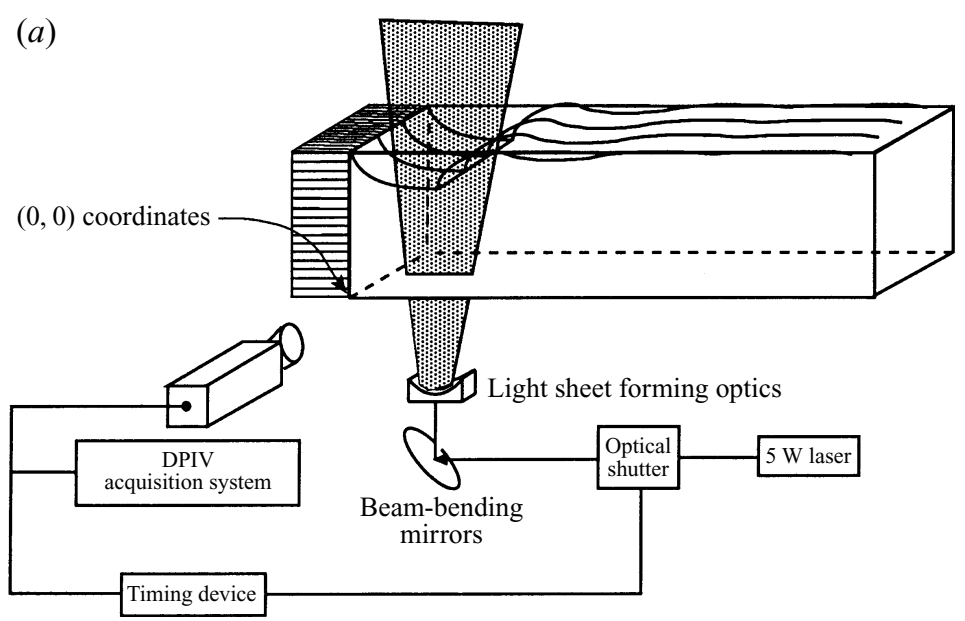

(b)

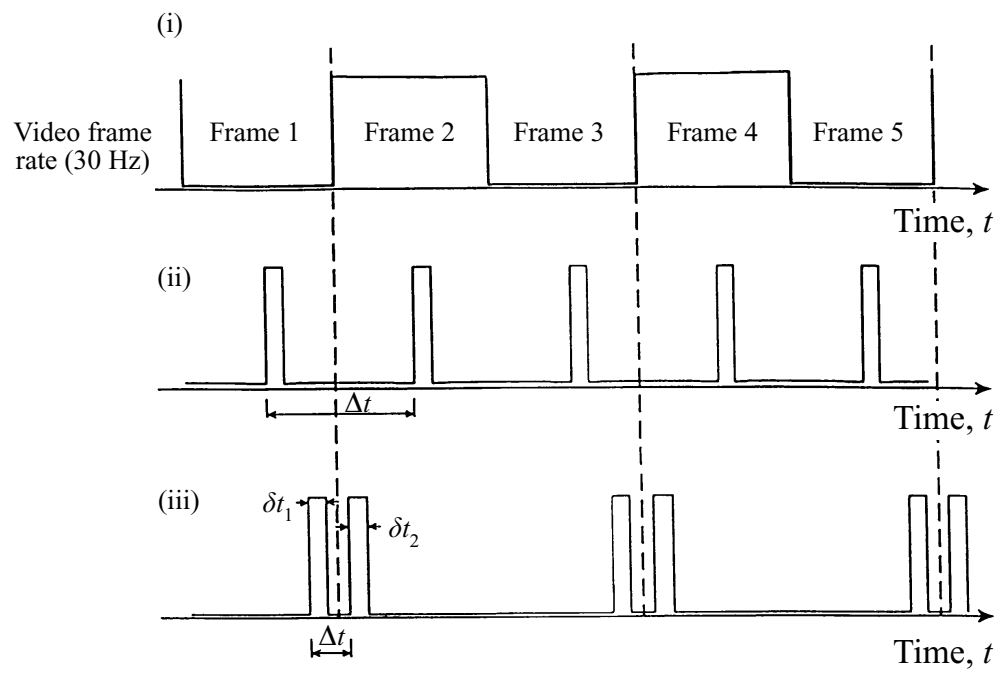

FiguRE 2. (a) Image acquisition set-up. (b) High-speed cross-correlation DPIV timing diagram in relation to conventional cross-correlation DPIV timing diagram. (i) The frame rate of the video camera, (ii) the pulsed exposure of CCD camera for typical cameras, (iii) the pulsed exposure capability of the camera.

\subsection{Free-surface treatment of the DPIV images}

The acquired images have reflections over the free surface that may affect the DPIV processing and result in erroneous velocity results. It is therefore imperative to treat the image area above the free surface. First, the image area above and including the free surface is replaced with a constant value equal to the image background of the fluid beneath the free surface. Then DPIV is performed on these images. Since the area above the free surface has no particles, the cross-correlation produces a zero shift. Interrogation windows which incorporate part of the image above the free surface and part below the free surface will therefore produce cross-correlation peaks that are due only to particle shifts below the free surface. For these windows, results are only considered valid when the window's centre is either at or below the free surface. If the vorticity were to be calculated from these vector fields, an artificial shear layer would 


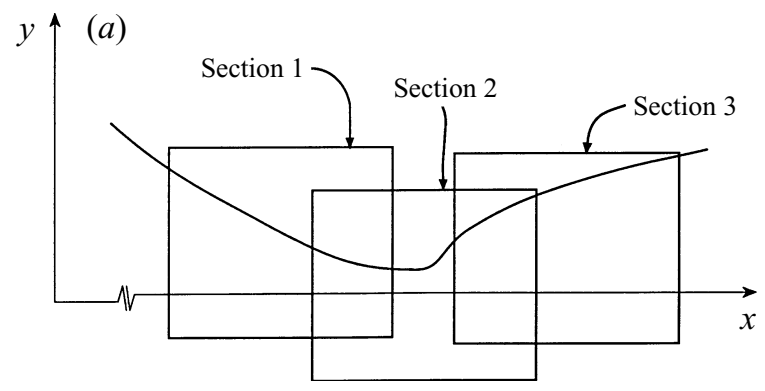

Section $1: 1.75<x<4.11 \mathrm{~cm} \quad 9.55<y<11.35 \mathrm{~cm}$ Section $2: 3.07<x<5.47 \mathrm{~cm} \quad 9.35<y<11.15 \mathrm{~cm}$ Section $3: 4.15<x<6.55 \mathrm{~cm} 10.40<y<12.20 \mathrm{~cm}$

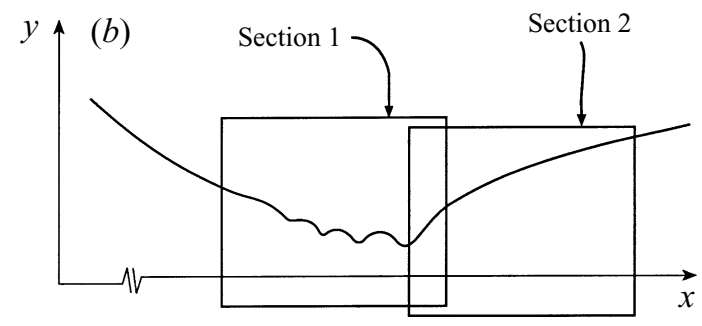

Section $1: 0.27<x<2.27 \mathrm{~cm} 10.40<y<13.03 \mathrm{~cm}$ Section $2: 1.31<x<3.21 \mathrm{~cm} 10.40<y<13.03 \mathrm{~cm}$

FIGURE 3. Imaged areas within a spilling wave (not to scale). (a) Higher Froude and Reynolds number case; $(b)$ lower Froude and Reynolds number case.

be generated at the free surface. Therefore, in order to minimize this effect, the no-shear boundary condition is applied at the free surface. All vectors above the last valid vector within a column are replaced by that vector for each column within the velocity field. Then vorticity is calculated. Since there are only velocity gradients in the $x$-direction above the free surface, only artificial vertical contours will be generated above the free surface in the vorticity plots, while accurate vorticity contours will be generated below the surface. Details concerning this approach will be published in a separate paper.

\section{Results and discussion}

Two sets of experiments were done in order to answer the questions posed in the Introduction. The first set was done at higher Reynolds and Froude numbers. Though very informative, this set of results produced capillary waves which were too small, and therefore it was not possible to ascertain the role of capillary waves in the flux of vorticity. Therefore a second set of experiments were done at lower Reynolds and Froude numbers to produce larger capillary waves, in order to determine their role in the vorticity flux.

\subsection{Case 1: higher Froude and Reynolds numbers}

For this case, the incoming velocity to the grid is $24.6 \mathrm{~cm} \mathrm{~s}^{-1}$. Owing to the pressure drop across the honeycomb/screen section, the fluid accelerates, while dropping in height by $4.2 \mathrm{~cm}$ (figure 1). Breaking occurs at the point when the fluid elevation sharply rises. The breaker rises to a maximum height of $1.1 \mathrm{~cm}$ (figure 1). Based on the velocity at the point immediately prior to breaking and the breaker height, the 

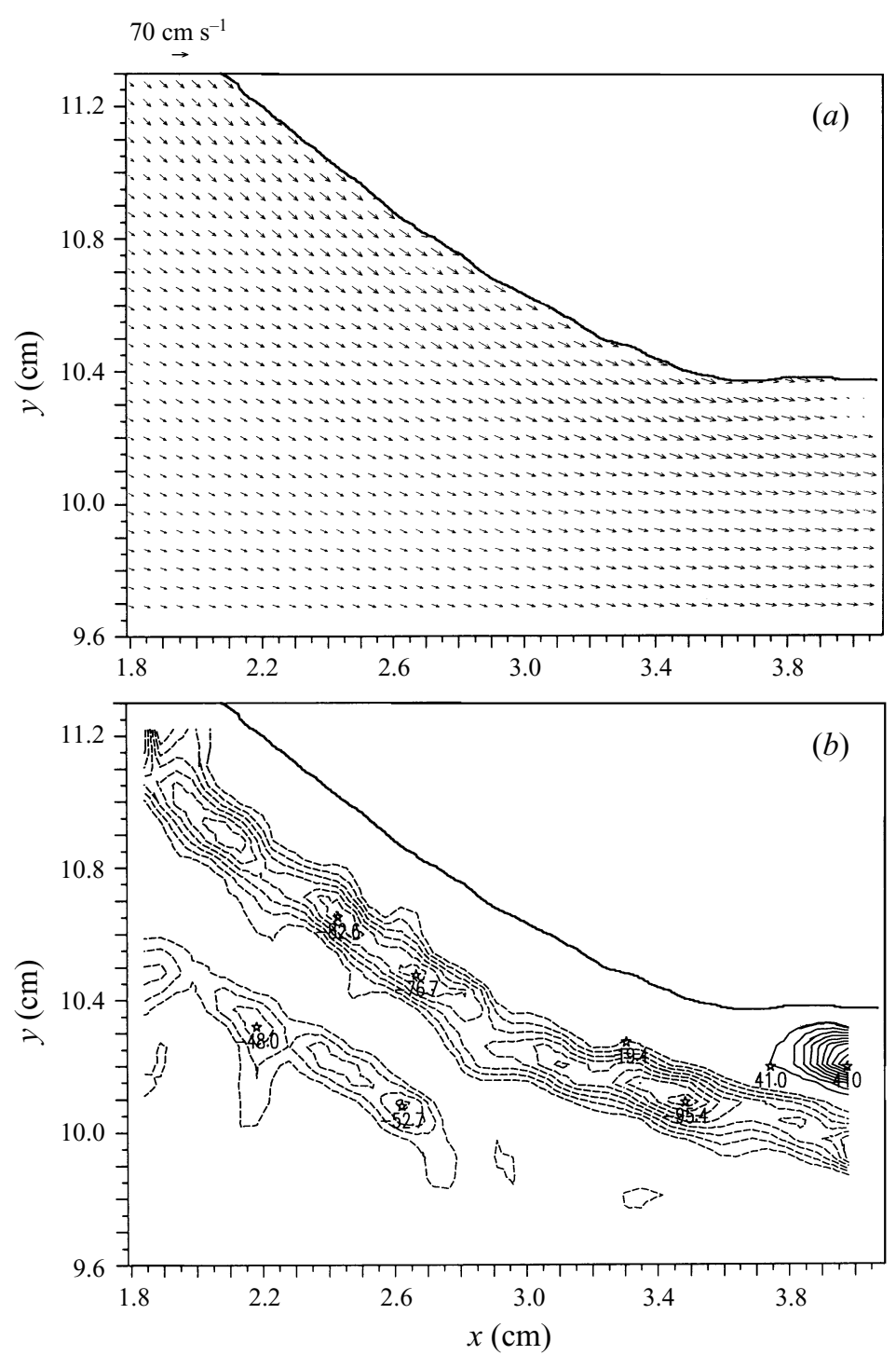

FIGURE 4. (a) Vector field of section 1 of case 1. (b) Vorticity field of section 1 of case 1. The negative contours (dashed lines) are in increments of $10 \mathrm{~s}^{-1}$ while the positive contours (solid lines) are in increments of $40 \mathrm{~s}^{-1}$.

Reynolds and Froude numbers are 7370 and 2.04, respectively. For this case, three regions, shown in figure $3(a)$, are interrogated. Section 1 interrogates the wave region where breaking occurs at the far right of the imaged area. Section 2 interrogates the wave where breaking occurs at the middle of the imaged area. Lastly, section 3 interrogates the wave where breaking occurs at the left of the imaged area.

\subsubsection{Velocity and vorticity fields}

The velocity and vorticity fields for section 1 are plotted in figure $4(a, b)$. The vectors within the velocity fields show the magnitude and direction of the velocity vectors throughout each of the three sections. For the vorticity plots, positive contours are shown by solid lines, while negative contours are shown by dashed lines. The velocity 


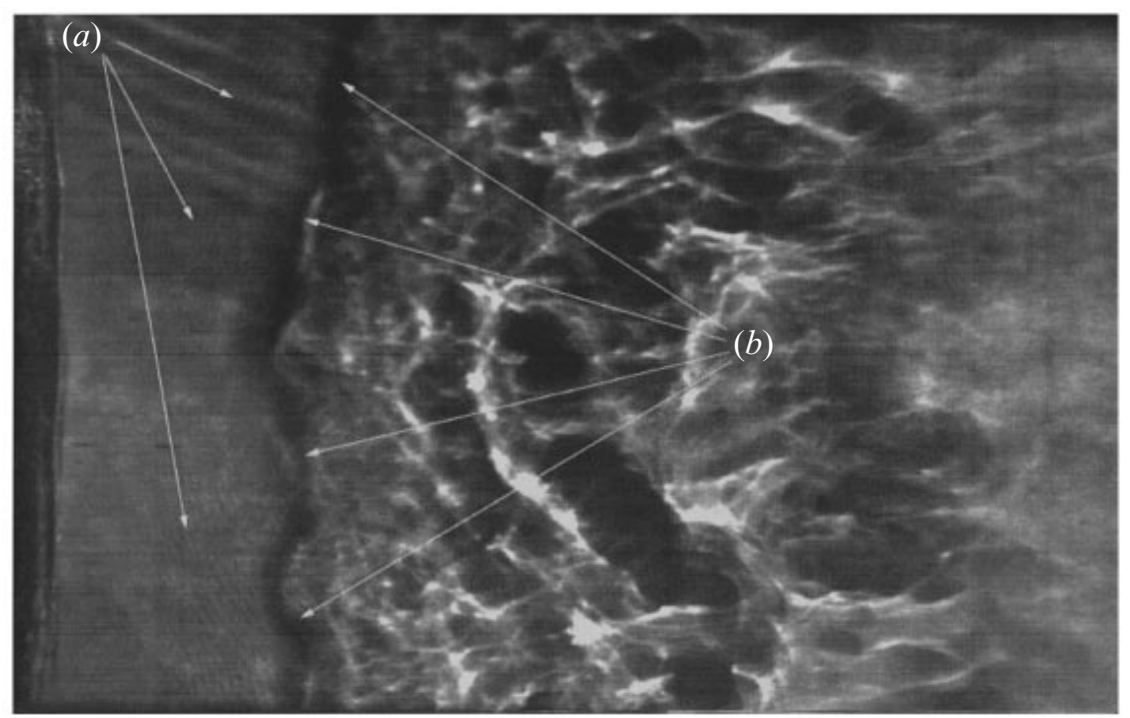

FIGURE 5. Shadowgraph for case 1. Flow is from left to right: $(a)$ shows the capillaries, while $(b)$ shows the boundary at which wave breaking starts.

field shows high velocities, except for a small region just beneath the free surface at $4.0 \mathrm{~cm}$ downstream from the grid, where the velocities are almost negligible. The vorticity field shows that the fluid contains mostly negative vorticity except for a small region just below the surface between 3.7 to $4.0 \mathrm{~cm}$ which contains positive vorticity. Upstream from this positive vorticity, the fluid directly beneath the free surface for a depth of $0.2 \mathrm{~cm}$ is relatively vorticity-free. Furthermore, the vorticity field also shows the existence of a negative vorticity layer located $0.3 \mathrm{~cm}$ below the free surface, which exists directly below the vorticity-free layer, and has a thickness of $\sim 0.3 \mathrm{~cm}$. The negative vorticity layer starts upstream, extends downstream to the positive vorticity region located at $3.68 \mathrm{~cm}$, and continues further downstream, but below the positive vorticity layer. The capillaries preceding spilling wave breaking are $\leqslant 1 \mathrm{~mm}$ in wavelength, and are too small to produce results that clearly determine the role of the capillaries in vorticity production. However, the raw images do show that the amplitude (crest-to-trough height) to wavelength ratio is $\sim 0.18$. The calculated phase speed of these capillaries is $68.7 \mathrm{~cm} \mathrm{~s}^{-1}$, and matches the velocity just prior to wave breaking (Cox 1958; Longuet-Higgins 1963).

A shadowgraph for section 2 is shown in figure 5, where the flow is from left to right. The capillary waves preceding wave breaking are clearly seen and are labelled $(a)$. The beginning of breaking is shown by the line labelled $(b)$, and finally spilling breaking is seen to the right of $(b)$. The shadowgraph shows that the breaker free surface has smallscale deformations on the free surface. This is due to the turbulent zone directly beneath the breaker, the existence of which will be shown below.

Figures $6(a, b)$ and $7(a, b)$ show the velocity and vorticity fields at section 2 for two different times, with a time difference of $0.267 \mathrm{~s}$. The vector plots show that directly beneath the breaker the velocities are quite small with respect to the rest of the flow. Furthermore, note that for both these cases, the high-speed free-surface layers directly beneath the free surface and upstream of the positive vorticity layer are also evident. Also, the negative vorticity layer, which was shown to exist beneath the free-surface high-speed layer in section 1, still continues further downstream beneath the positive 

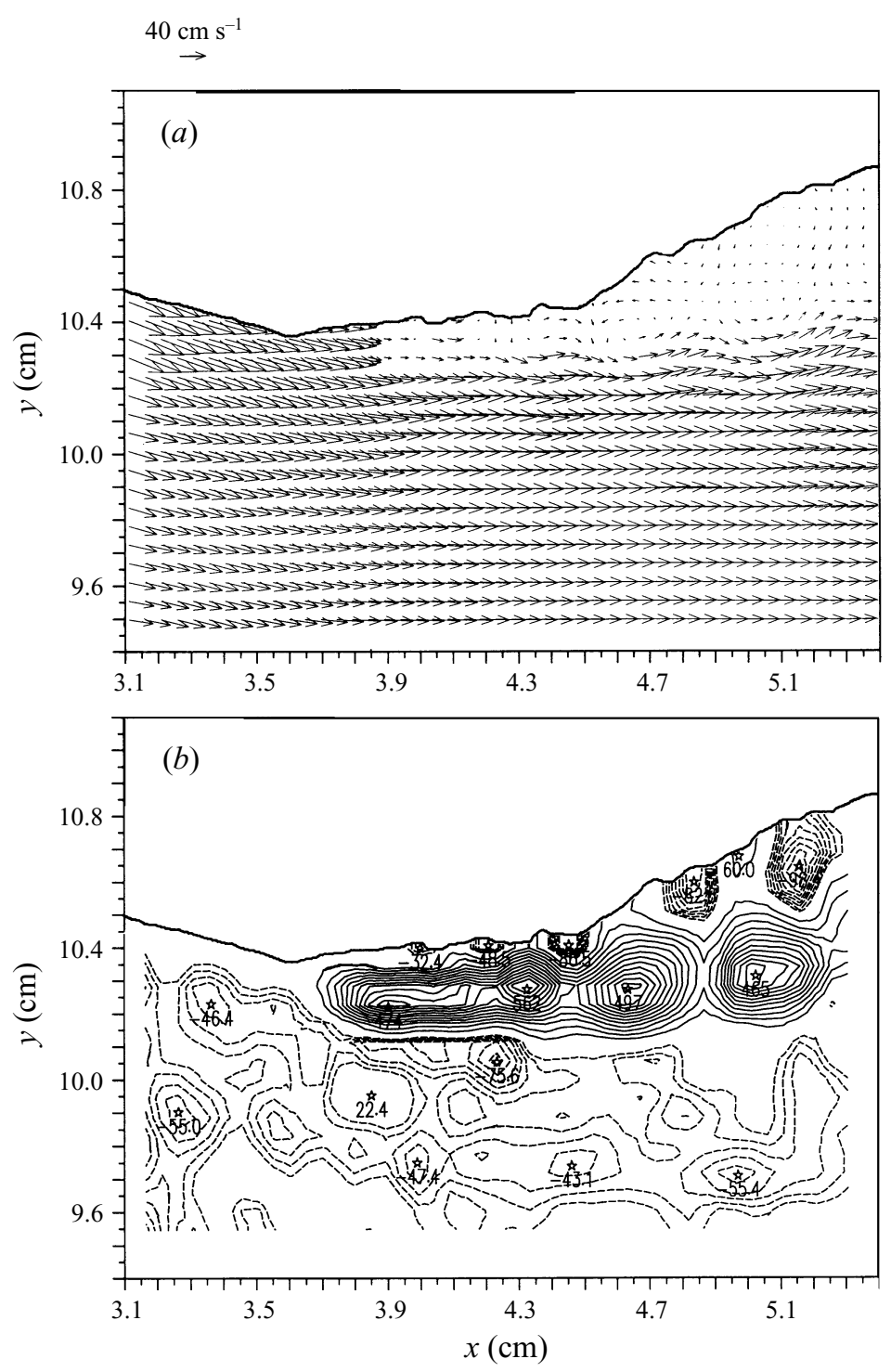

Figure 6. (a) Vector field of section 2 of case 1. (b) Vorticity field of section 2 of case 1. Contours as for figure $4(b)$.

vorticity layer. Also, the fully developed positive vorticity layer which was initially seen in section 1 is captured in figures $6(b)$ and $7(b)$, and seen to convect downstream. This positive vorticity layer, however, does not separate into a shear layer until the free surface sharply curves upwards. Note that there are also areas of negative vorticity at the free surface that are not as strong in magnitude as those in the mixing layer. A comparison of the flow of figures $6(b)$ and $7(b)$, shows that the positive vorticity shear layer origin has been displaced by $0.5 \mathrm{~cm}$ in the $x$-direction. In fact, after examining several minutes of the flow in detail, it becomes evident that this 'breathing' effect is quite persistent yet erratic in amplitude, with an oscillation frequency of $\sim 3.75 \mathrm{~Hz}$. It was initially believed that this frequency might be the shedding frequency. However, it can be shown that this is not the case. Figure $6(b)$ shows the necessary parameters to determine this frequency. The convective velocity of the rollers in the shear layer is 

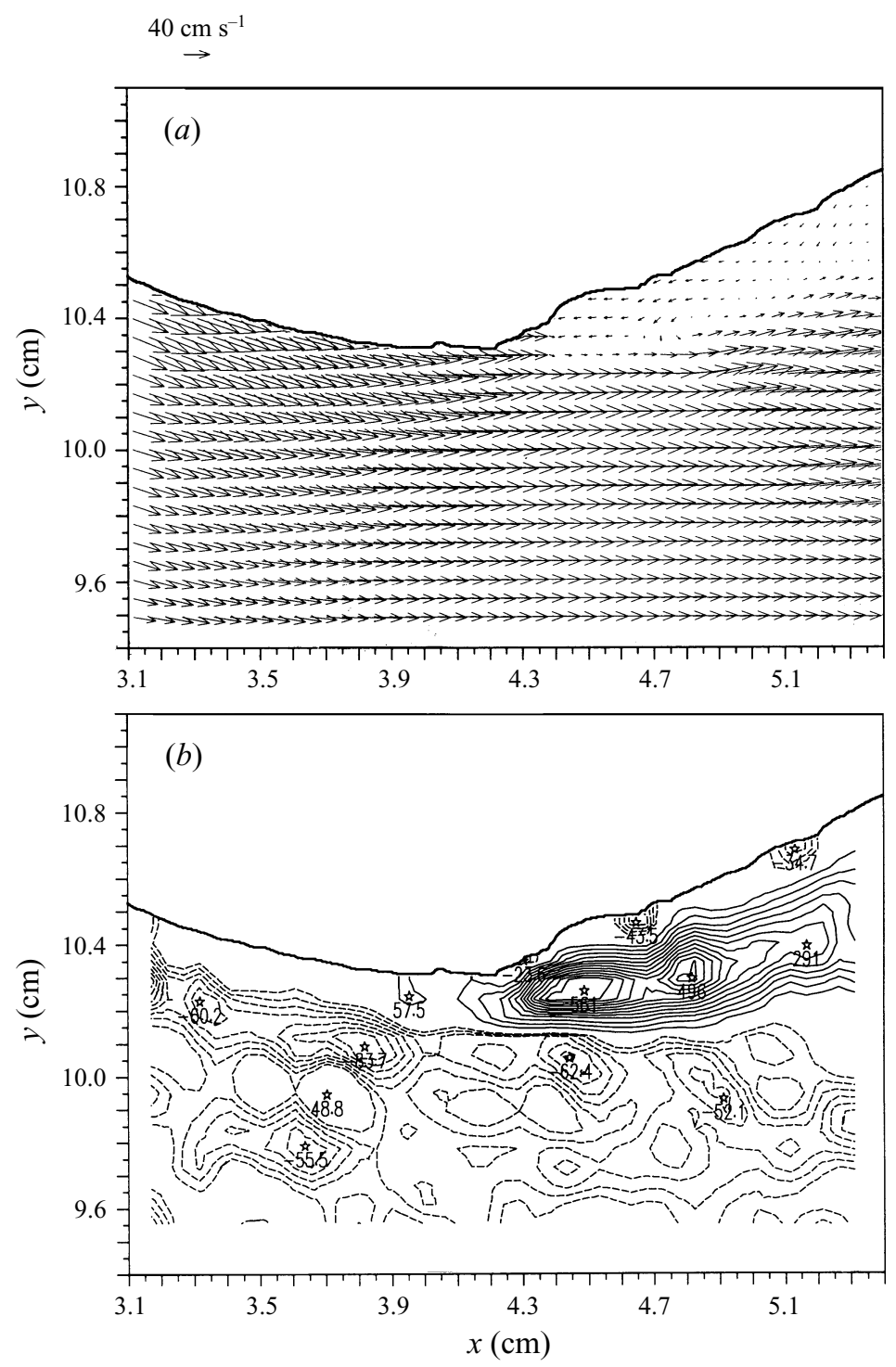

FIGURE 7. (a) Vector field of section 2 of case $10.267 \mathrm{~s}$ after figure $6(a)$. (b) Vorticity field of section 2 of case $10.267 \mathrm{~s}$ after figure $6(b)$. Contours as for figure $4(b)$.

approximately the average of the velocity across the shear layer, or $\sim 33.5 \mathrm{~cm} \mathrm{~s}^{-1}$. The average wavelength between the rollers is $0.35 \mathrm{~cm}$, resulting in a shedding frequency of $\left(33.5\left(\mathrm{cms}^{-1}\right) / 0.35 \mathrm{~cm}\right) \sim 96 \mathrm{~Hz}$. The maximum vorticity within the positive vorticity layer shown in figure $7(b)$ is $652 \mathrm{~s}^{-1}$. Therefore, the dimensionless maximum vorticity is $w_{\max } \lambda_{\max } / U=2.94$, where $U$ is the free-surface velocity prior to breaking.

Finally, figure $8(a, b)$ shows the velocity and vorticity field associated with section 3 . The vorticity field shows the persistence of the shear layer beneath the breaker. It is also interesting to note that even though the flow between the shear layer and the free surface is turbulent, its vorticity field is quite weak with respect to the vorticity within the shear layer. This is consistent with the observations of Lin \& Rockwell.

Of interest is the existence of a thin layer of almost vorticity-free fluid just beneath the surface prior to wave breaking. To further examine this region, velocity profiles 

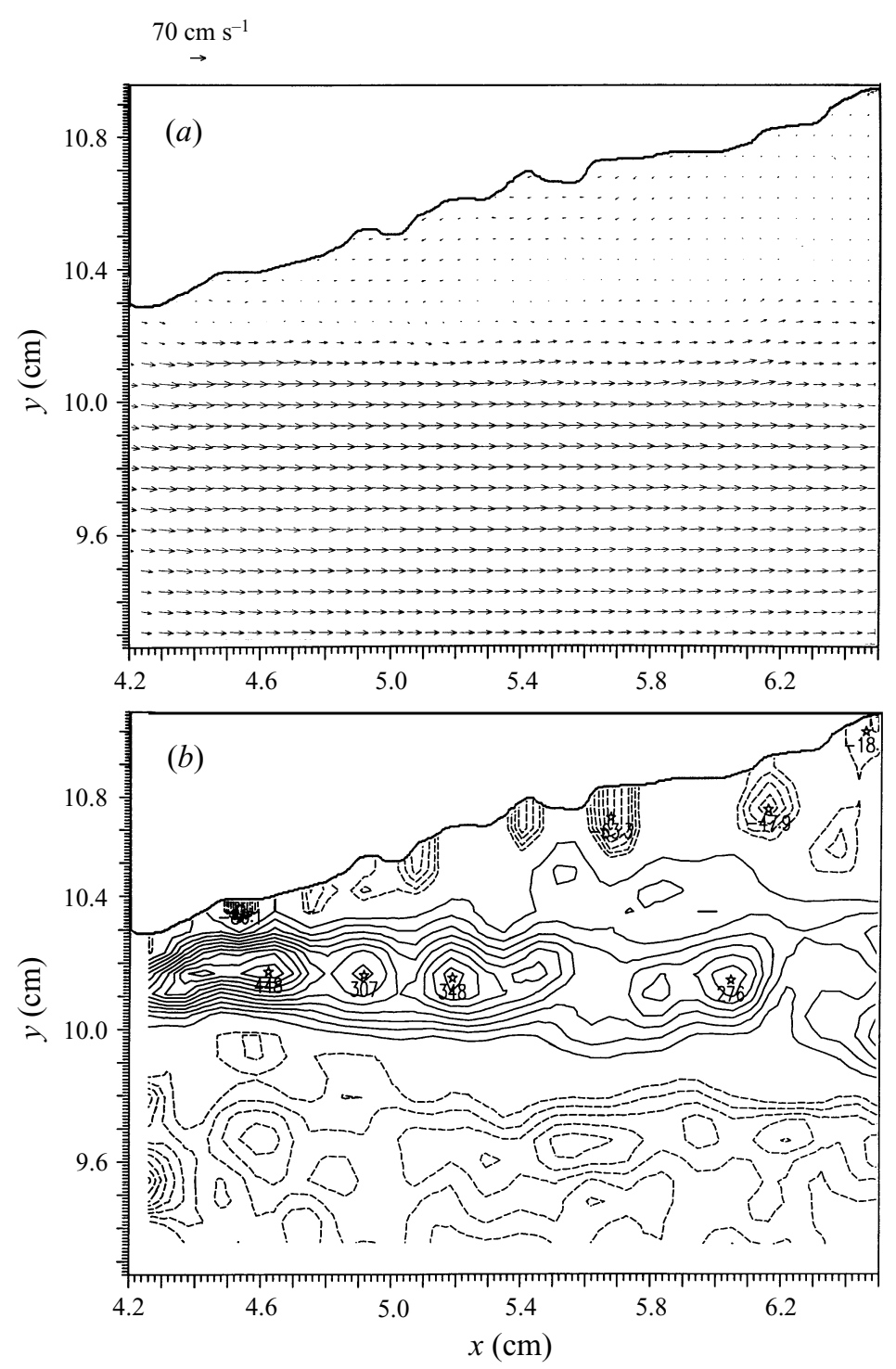

FIGURE 8. (a) Vector field of section 3 of case 1. (b) Vorticity field of section 3 of case 1. Contours as for figure $4(b)$.

perpendicular to the free surface are extracted at various locations, and plotted in figure 9. These plots show that at the free surface there is a thin layer of fluid that accelerates with respect to the rest of the fluid beneath it. Examination of previous work using different wave breaking generation techniques did not reveal the existence of a high-speed free-surface layer, suggesting that this layer is particular to our method of wave breaking. This layer originates from the free surface fluid immediately after the honeycomb/screen section. As the flow goes through the honeycomb/screen section, it will feel a pressure drop. For free-surface flows, this pressure drop is seen as a drop in height, as well as an acceleration of the fluid. At the same time some turbulence will be generated. However, on the free surface, due to the free-shear boundary condition, the free surface does not support any shear. As such, a thin free-surface layer will 

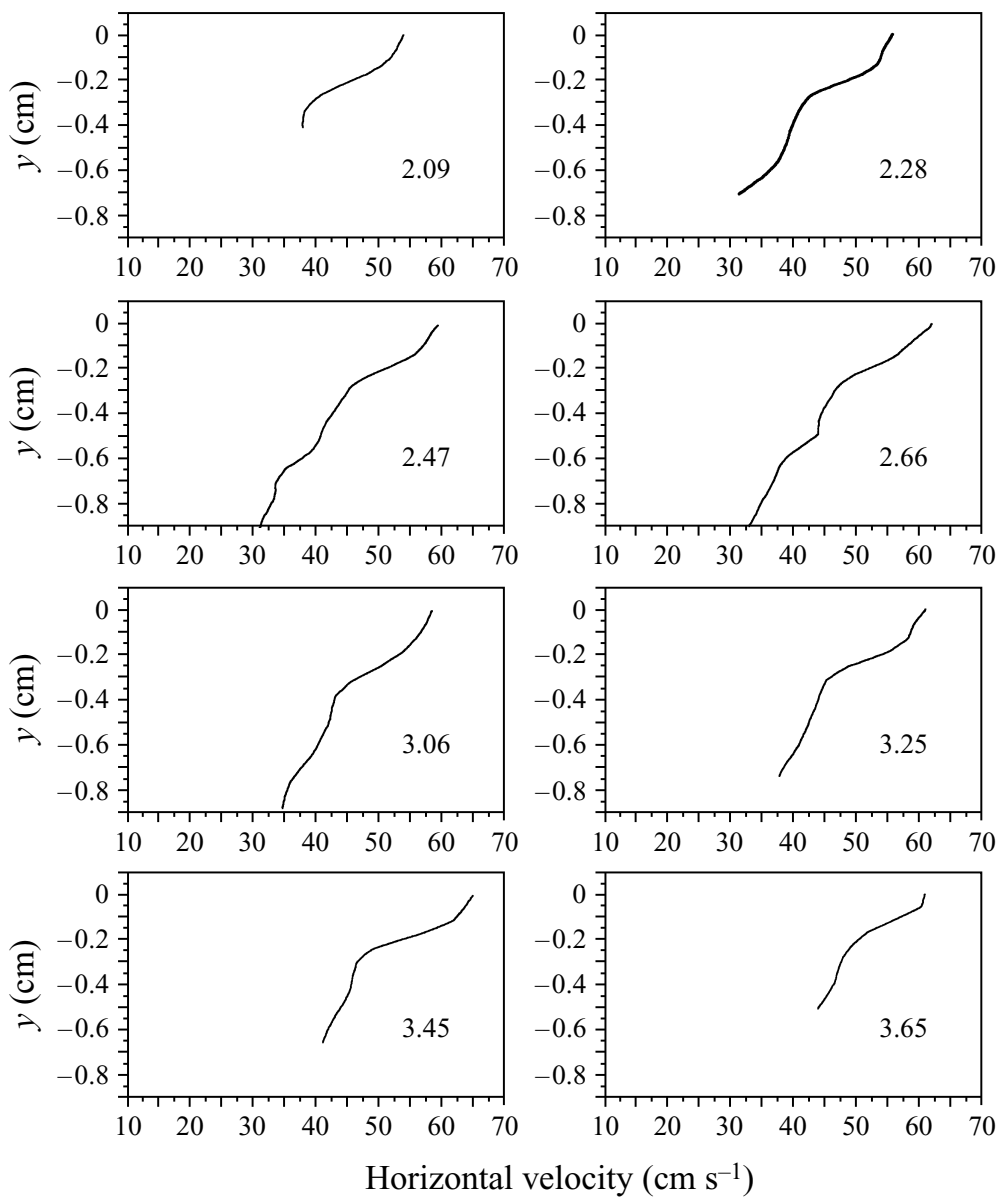

FIGURE 9. Case 1: velocity profiles perpendicular to the free surface extracted from various points through the free surface. The number on each plot indicates the $x$-axis location of the free surface through which the profile passes.

accelerate faster than the fluid below it, thereby generating a higher-velocity freesurface layer. The velocity and vorticity plots in figure $4(a, b)$ show that this layer rapidly decelerates at $\sim 3.9 \mathrm{~cm}$, immediately after which positive vorticity is seen. This process seen at the free surface seems to be independent of the flow beneath it, since the negative vorticity layer beneath the free-surface high-speed layer and the positive vorticity shear layer isolate that region from the bulk of the flow. Consequently, the flow locally beneath the surface and above the negative vorticity shear layer is reminiscent of a hydraulic jump. In this respect, the appropriate Froude number should be based on the velocity within the free-surface higher-velocity layer and thickness of this layer. For this case, the corresponding velocity and layer thickness are approximately $67 \mathrm{~cm} \mathrm{~s}^{-1}$ and $0.2 \mathrm{~cm}$, resulting in a perhaps more accurate and descriptive incoming Froude number of 4.78. Taking this a step further, the hydraulic jump equations can be used to verify whether or not the phenomenon seen can be described as a hydraulic jump. The hydraulic jump height relation is

$$
\frac{h_{2}}{h_{1}}=\frac{1}{2}\left[-1+\left(1+8 F r_{1}^{2}\right)^{1 / 2}\right]
$$




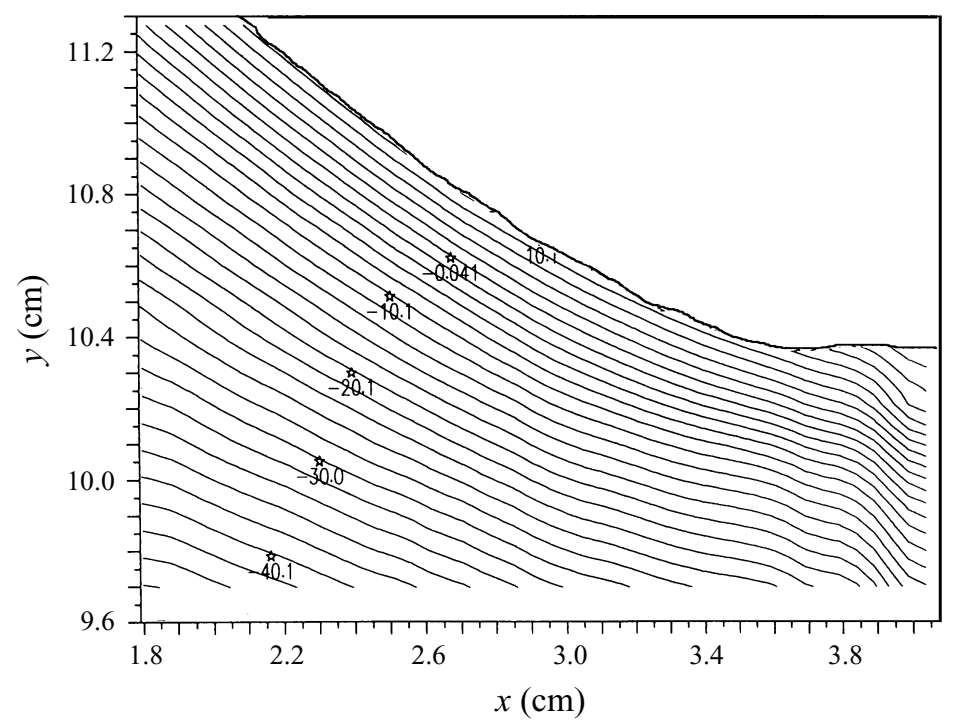

FIGURE 10. Streamline plot of section 1 of case 1 . Contours are in increments of $2 \mathrm{~cm}^{2} \mathrm{~s}^{-1}$.

where $h_{1}$ and $F r_{1}$ are the pre-jump fluid height and Froude number, respectively, and $h_{2}$ is the post-jump fluid height. This equation can be used to predict the height of the jump. Using $h_{1}=0.2 \mathrm{~cm}$, and $F r_{1}=4.78, h_{2}=1.26 \mathrm{~cm}$. Subtracting the pre-jump height gives a breaker height of $1.06 \mathrm{~cm}$, which agrees quite nicely with our initial breaker height measurement of $1.1 \mathrm{~cm}$. Before concluding that this phenomenon can be modelled as a hydraulic jump, the assumptions that are imposed in the derivation of equation (1) should be tested. The first assumption is that the pressure within the hydraulic jump is hydrostatic, which we will also take as an assumption. According to the second assumption, the classical hydraulic jump derivation assumes that the viscous force exerted by the horizontal bottom is negligible in comparison to local pressure forces. It can be shown (Thompson 1972) that the one-dimensional, shallow water expression for this condition is

$$
\frac{\partial h}{\partial x} \gg \frac{F r_{h}^{2}}{R e_{h}} \text { or }\left(\frac{F r_{h}^{2}}{R e_{h}} / \frac{\partial h}{\partial x}\right) \ll 1
$$

where $R e_{h}=h u / v$, is the height of the hydraulic jump, and $x$ is the direction of the flow. For our case, there is no solid boundary. We therefore choose an internal streamline to be the lower boundary. Figures 10, 11 and 12 show the streamlines for sections 1 , 2 and 3 mentioned above. Note that the evenly spaced streamlines just below the surface have been vertically displaced by $0.2 \mathrm{~cm}$ at the right-hand part of figure 10 . This is also confirmed by the streamlines shown in figure 11, where the first streamline parallel to the free surface with a value of $-15 \mathrm{~cm}^{2} \mathrm{~s}^{-1}$ is $0.2 \mathrm{~cm}$ below the free surface. We therefore choose the streamline at $0.2 \mathrm{~cm}$ below the free surface as the lower boundary. Using this depth as the length scale, $R e_{h}=1340$, and $F r_{h}=4.78$. Figure 12 shows that $\partial h / \partial x \approx 0.3$. Therefore, condition (2) results in

$$
\left(\frac{F r_{h}^{2}}{R e_{h}}\left(\frac{\partial h}{\partial x}\right)=5.7 \times 10^{-2} \ll 1\right.
$$




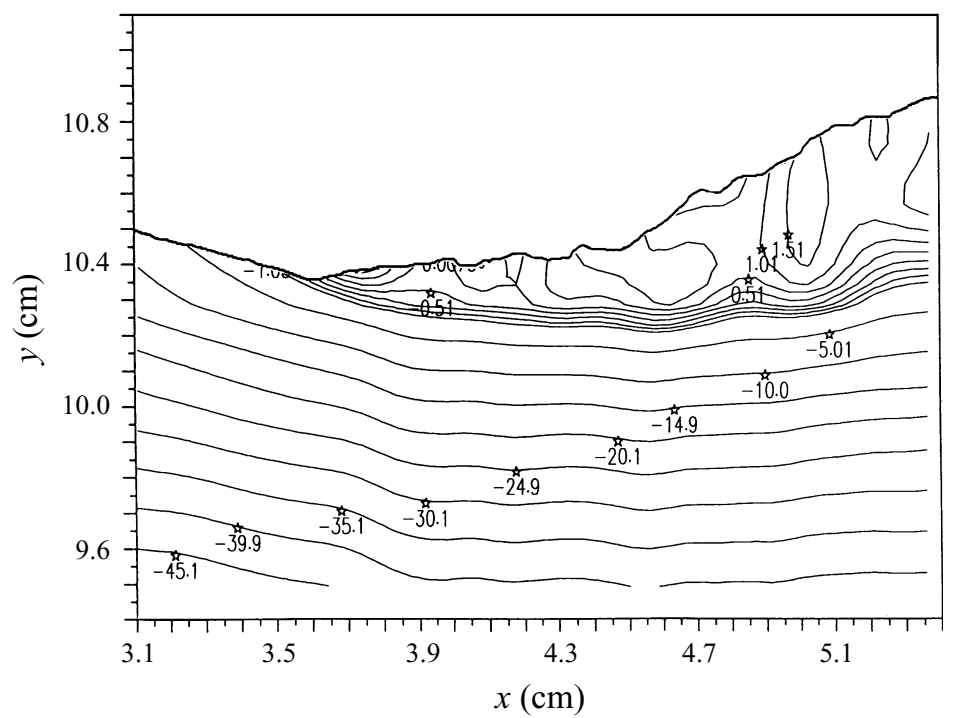

FIGURE 11. Streamline plot of section 2 of case 1 . Contours between the values of -50 and $-5 \mathrm{~cm}^{2} \mathrm{~s}^{-1}$ are in increments of $5 \mathrm{~cm}^{2} \mathrm{~s}^{-1}$, those between -2 and $2 \mathrm{~cm}^{2} \mathrm{~s}^{-1}$ are in increments of $0.5 \mathrm{~cm}^{2} \mathrm{~s}^{-1}$.

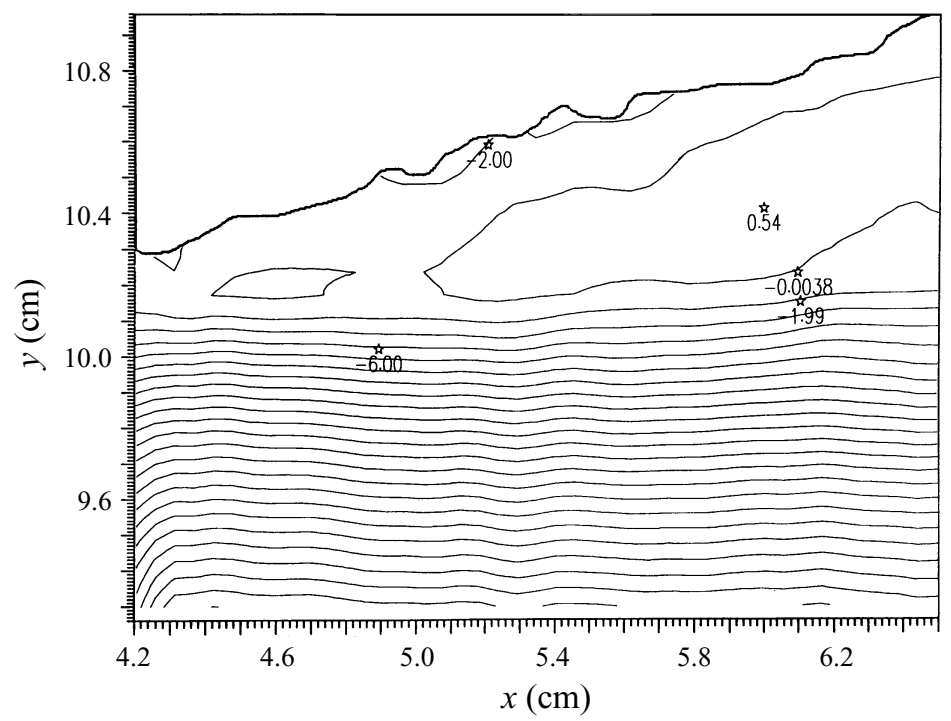

FIGURE 12. Streamline plot of section 3 of case 1 . Contours are in increments of $2 \mathrm{~cm}^{2} \mathrm{~s}^{-1}$.

Clearly this condition is satisfied, indicating that the spilling breaker can be modelled as a local hydraulic jump.

\subsubsection{Sources of vorticity}

As has been seen by the previous investigators and shown in the present velocity and vorticity fields, upon breaking irrotational flow becomes highly rotational. In order to understand the origin of this vorticity, one must first look at the terms which contribute 


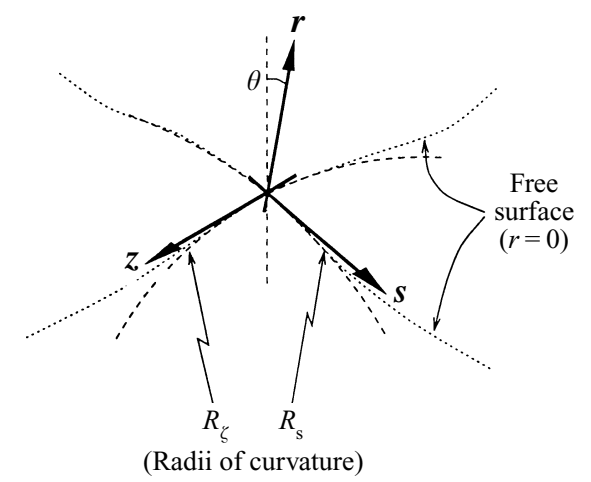

FIGURE 13. The natural curvilinear orthogonal coordinates: $\boldsymbol{r}$ is the unit vector perpendicular to the free surface, $s$ is the unit vector parallel to the free surface, and perpendicular to $r$, and $z$ is the unit vector parallel to the free surface and perpendicular to $s$ and $r$.

to sources of vorticity. The vorticity flux of surface-parallel vorticity through the surface given in natural curvilinear orthogonal coordinates is (Rood 1994, 1995; Gharib \& Weigand 1996)

$$
v\left(\frac{\partial \omega_{z}}{\partial r}\right)_{r=0}=-\frac{\partial u_{s}}{\partial t}-\frac{1}{\rho} \frac{\partial P}{\partial s}-g \cos \theta-\frac{1}{2} \frac{\partial u_{s}^{2}}{\partial s}
$$

where $v$ is the kinematic viscosity, $\omega_{z}$ is the surface-parallel vorticity, $u_{s}$ is the velocity parallel to the free surface, $u_{r}$ is the velocity normal to the free surface, $\rho$ is the fluid density, $P$ is the pressure, and $\theta$ is the angle of the surface with respect to the gravity vector. Figure 13 shows the system of coordinates used in equation (3). For the steady breaker,

through time. Therefore,

$$
u_{s=0} \approx \text { constant }
$$

$$
\frac{\partial u_{s}}{\partial t} \approx 0
$$

Likewise, the pressure along the free surface is constant. Therefore,

Furthermore,

$$
\frac{\partial P}{\partial s} \approx 0
$$

$$
|g \cos \theta| \leqslant|g|=981 \mathrm{~cm} \mathrm{~s}^{-2} .
$$

Therefore, the dominant terms contributing to the velocity flux are the gravity term and the deceleration term. Equation (3) thus reduces to

$$
v\left(\frac{\partial \omega_{z}}{\partial r}\right)_{r=0} \approx-g \cos \theta-u_{s} \frac{\partial u_{s}}{\partial s}
$$

\subsubsection{Experimental observations of the sources of vorticity}

In order to understand the behaviour of the vorticity flux, one must first examine the behaviour of the deceleration and the gravity terms. Figure 14 shows the deceleration and the surface velocity for section 1 . The deceleration shows a strong peak at $3.84 \mathrm{~cm}$ 


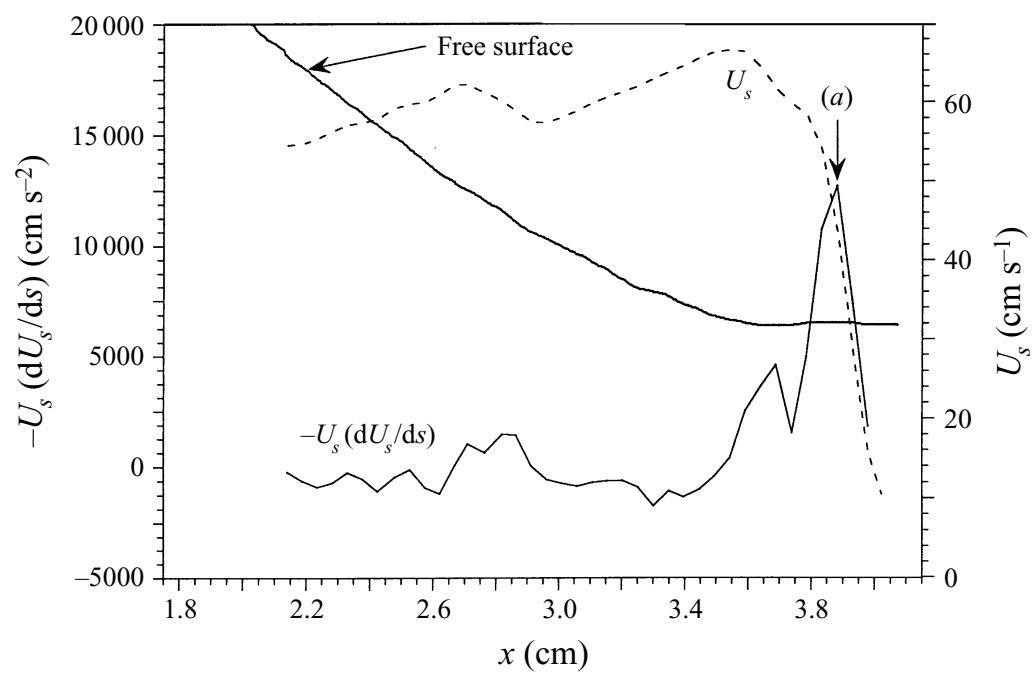

FIGURE 14. The free-surface velocity and deceleration for section 1 of the higher Froude number case. The vorticity flux is shown on the left-hand axis, while the velocity scale is shown on the right-hand axis; (a) at $3.84 \mathrm{~cm}$ shows the position of the maximum deceleration, and therefore flux of vorticity into the flow.

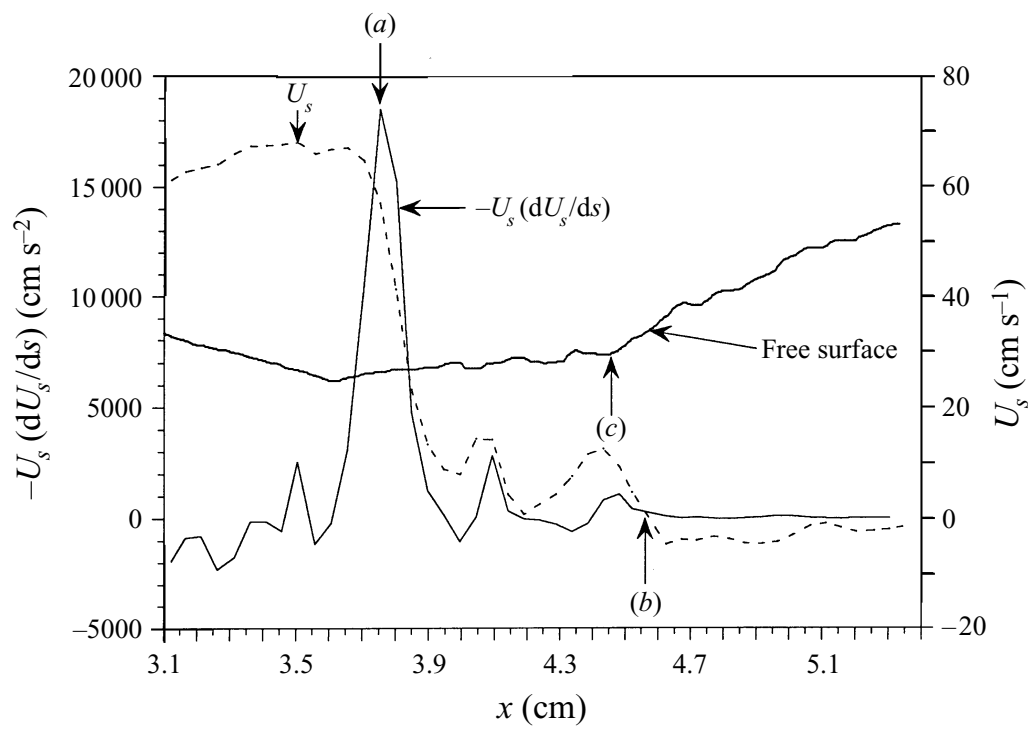

FIGURE 15. As figure 14 but for section 2: (a) at $3.75 \mathrm{~cm}$ shows the position of the maximum deceleration, and therefore flux of vorticity into the flow; $(b)$ at $4.58 \mathrm{~cm}$ shows the stagnation point of the free-surface fluid, and $(c)$ at $4.47 \mathrm{~cm}$ shows initiation of wave breaking observed visually.

from the origin. This coincides with the strong generation of positive vorticity seen in figure 4(b). The velocity curve shows that the velocity rapidly decreases after $3.5 \mathrm{~cm}$ from the origin. However, the fluid does not approach a stagnation point until at least $0.15 \mathrm{~cm}$ farther downstream from the peak. Figure 15 shows the deceleration and the surface velocity for section 2 . The deceleration reaches a peak at $3.75 \mathrm{~cm}$ from the origin, shown by $(a)$. This coincides with the strong generation of positive vorticity seen in figure $7(b)$. The free-surface velocity curve shows that the fluid reaches a stagnation 


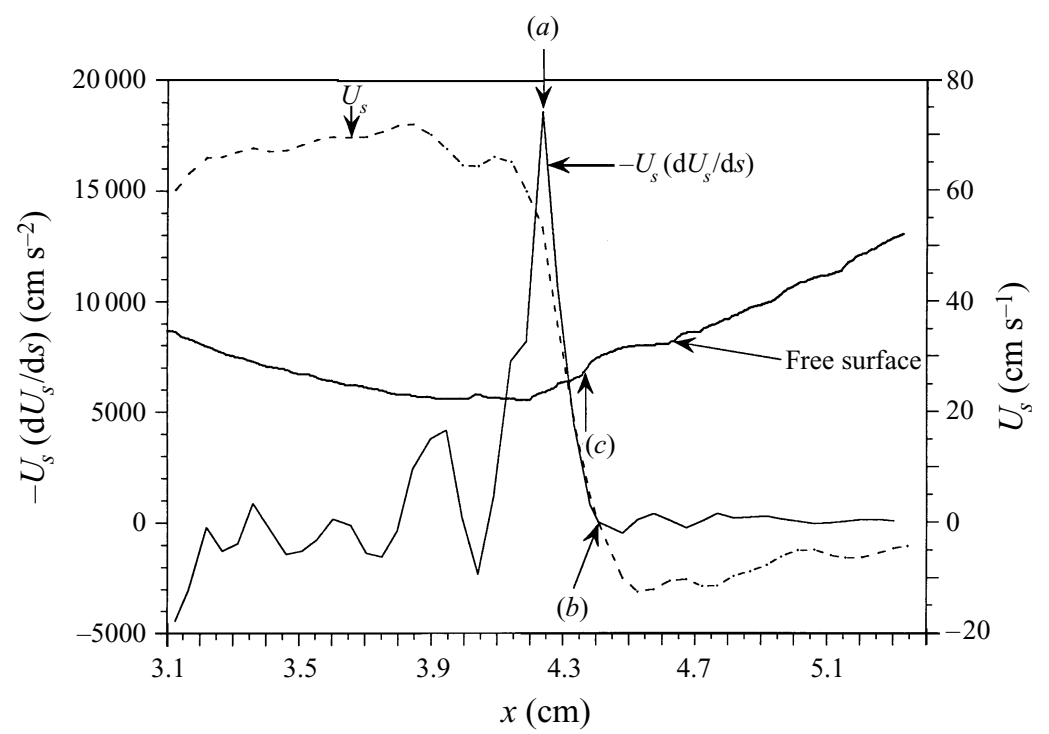

FIGURE 16. The free-surface velocity and deceleration for section $20.267 \mathrm{~s}$ after that shown in figure 15: (a) at $4.25 \mathrm{~cm}$ shows the position of the maximum deceleration, and therefore flux of vorticity into the flow; $(b)$ at $4.4 \mathrm{~cm}$ shows the stagnation point of the free-surface fluid, and $(c)$ at $4.35 \mathrm{~cm}$ shows initiation of wave breaking observed visually.

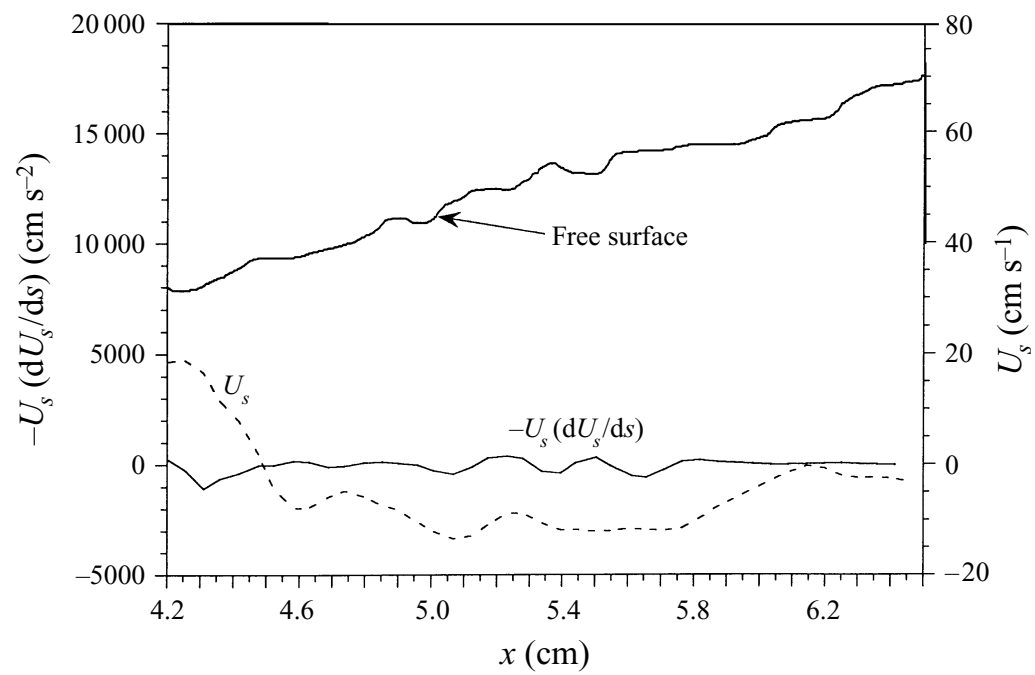

FIGURE 17. The free-surface velocity and deceleration for section 3 for the higher Froude number case. Note that in this case, there are no outstanding peaks, since there are no fluxes of vorticity at this section.

point at $4.58 \mathrm{~cm}$ shown by $(b), 0.83 \mathrm{~cm}$ downstream from the vorticity flux peak. Consequently, it is important to realize that the generation of vorticity does not coincide with the stagnation point. Furthermore, the initiation of wave breaking does not contribute to the positive vorticity generation. This can be seen by observing that the vorticity is generated $3.75 \mathrm{~cm}$ from the honeycomb/screen section, while visually observed breaking occurs at $4.47 \mathrm{~cm}$, shown by $(c)$. Figure 16 shows the deceleration and the surface velocity for section 2 at $0.267 \mathrm{~s}$ later than figure 15 . The deceleration 

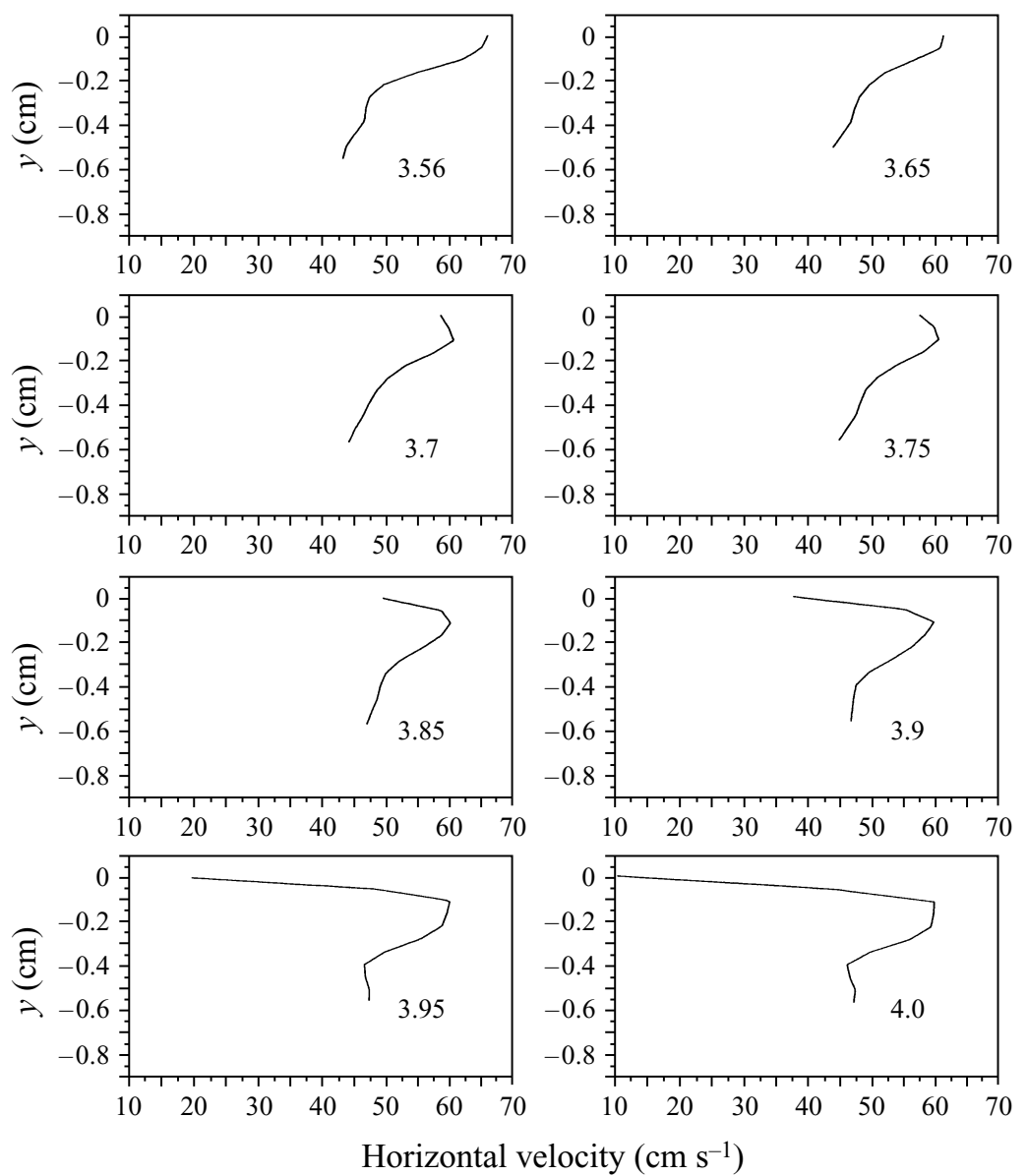

FIGURE 18. Velocity profiles taken from section 1 for case 1 at different $x$-locations. The profiles are perpendicular to the free surface, and the numbers are $x$-locations of the free surface. The origin on the $y$-axis indicates the free surface.

reaches its peak at $4.25 \mathrm{~cm}$ from the origin, shown by $(a)$, while the fluid stagnates at $4.4 \mathrm{~cm}$, shown by $(b)$. Therefore, as in the previous figure, the strong generation of vorticity represented by the deceleration of the free-surface fluid coincides with the appearance of strong positive vorticity. Curiously, figure 16 seems to indicate that the source of vorticity might be the sharp curvature of the free surface due to breaking, shown by $(c)$ at 4.35 , since vorticity generation and the sharp free-surface curvature coincide. However, this coincidence is due solely to the 'breathing' effect mentioned earlier where the positive vorticity layer oscillates at a lower frequency to and from the sharp free-surface curvature. Once again, it should be emphasized that figure 15 clearly shows that the source of vorticity is not due to the free-surface's sharp curvature, but due to the deceleration term shown in equation (8). It should be pointed out, however, that perhaps the best indication of breaking is the occurrence of the stagnation points shown by markers $(b)$ in figures 15 and 16 . For both these cases, even though the vorticity flux peaks fluctuate back and forth with respect to breaking, the stagnation points are consistently within $0.1 \mathrm{~cm}$ of breaking, thus verifying the observations of Banner \& Phillips. Finally figure 17 shows the deceleration and the velocity profiles for section 3. This plot does not show any of the strong peaks seen in the previous figures, 


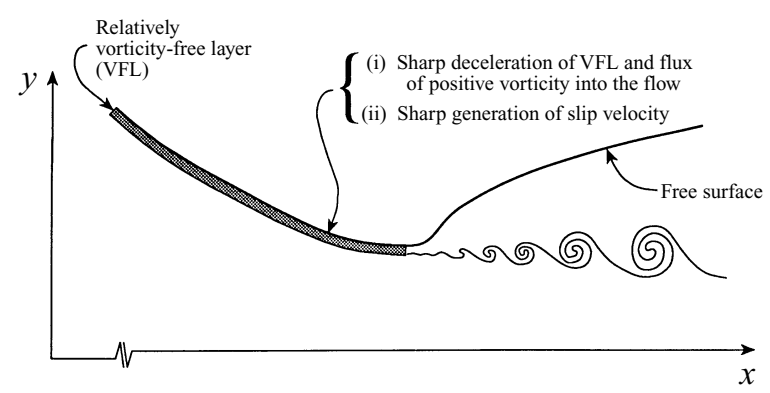

FIGURE 19. Diagram demonstrating the process of flux of vorticity into the flow. The vorticity-free layer decelerates sharply, introducing a high flux of vorticity into the flow, and simultaneously producing a sharp slip velocity which then grows into the shear layer seen beneath spilling breaker waves. (Drawing not to scale.)

since vorticity is not generated, but rather convected downstream. Thus, the important point to realize in figures $14-16$ is that, given equation (8), the dominant contributing term to the velocity flux is the deceleration term, which is of the order of $20000 \mathrm{~cm} \mathrm{~s}^{-2}$, while the gravity term, which can be no greater than $981 \mathrm{~cm} \mathrm{~s}^{-2}$, is negligible with respect to the deceleration term.

Up to now, it has been shown that the flux of vorticity into the flow is due primarily to viscous effects. However, it can be argued that perhaps the vorticity flux is due to the velocity difference across the shear layer, thus making the vorticity flux an inviscid effect. Further reflection can resolve this issue. The reduced vorticity flux equation (8) derived earlier is shown below for convenience:

$$
\nu\left(\frac{\partial \omega_{z}}{\partial r}\right)_{r=0} \approx-g \cos \theta-u_{s} \frac{\partial u_{s}}{\partial s} .
$$

This equation, evaluated at the free surface, specifically shows that the source of vorticity is from the free surface, and that its flux from the free surface to the fluid, although equal to the free-surface fluid deceleration, is entirely a viscous process. However, the free-surface fluid not only decelerates with respect to the free-surface fluid upstream of itself, but also with respect to fluid directly beneath it, thus creating a sharp velocity gradient, or 'slip velocity'. Figure 18 shows several velocity profiles which are perpendicular to the free surface at section 1 of case 1, corresponding to figure $4(a, b)$. These velocity profiles are from various $x$-locations about $x=3.7 \mathrm{~cm}$, illustrating the large velocity gradient, or 'slip velocity' directly beneath the free surface. It is this 'slip velocity' that is the origin of the shear layer evident in figures $6(b), 7(b)$ and $8(b)$. It can therefore be concluded that the free-surface deceleration not only produces a vorticity flux into the flow through a diffusive process, but in doing so, simultaneously generates a sharp slip velocity which grows into a shear layer, convecting downstream the vorticity which is fluxed into the fluid from the free surface (see figure 19). This can also be seen by realizing that the free-surface fluid decelerates to a stagnation point. Therefore, the vorticity must be fluxed deeper into the fluid, beyond the stagnation region, so that it can be convected downstream through the shear layer. A good check of the above analysis is to first calculate the net vorticity flux from the free surface into the fluid bulk by integrating the fluid deceleration at the free surface; second, calculate the convected vorticity flux by integrating the vorticity flux in the $y$-direction for each $x$-location; and finally show that the vorticity fluxed into the flow is equal to the vorticity being convected through the flow. 


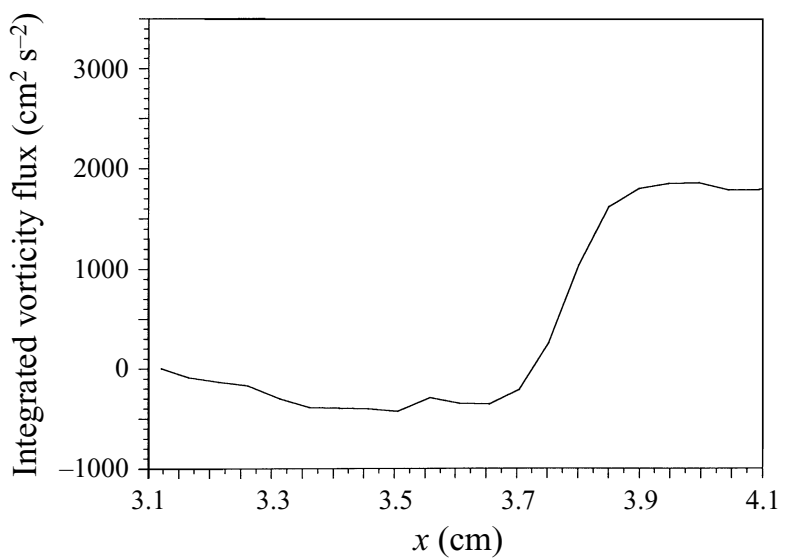

FIGURE 20. The running integral of the viscous vorticity flux of section 2 .

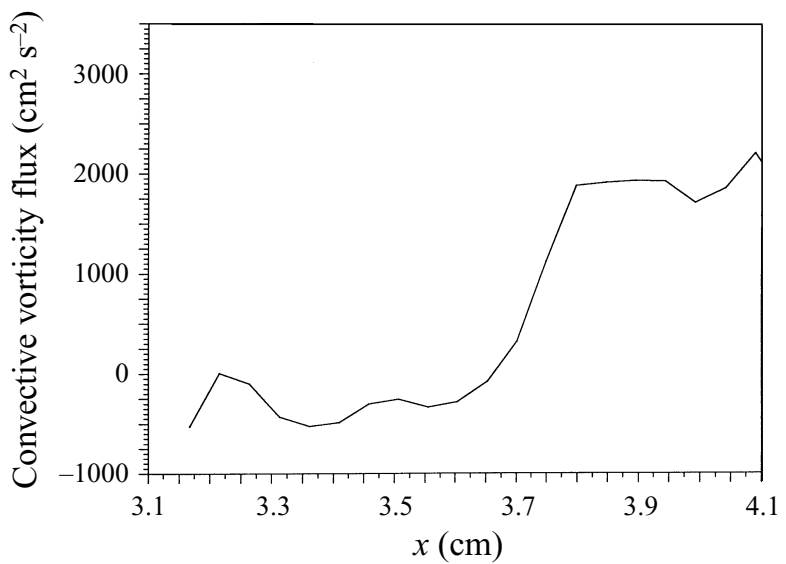

FIGURE 21. The integrated convective vorticity flux for section 2.

Figure 20 shows the integrated viscous vorticity for section 2, corresponding to figures $6(a, b)$ and 15 . Note that there is a sharp increase at $3.65 \mathrm{~cm}$ indicating a large injection of vorticity into the flow. The total increase is $\sim 2250 \mathrm{~cm}^{2} \mathrm{~s}^{-2}$. Beyond this injection, the level of integrated vorticity flux remains constant, indicating that there is no more vorticity flux from the free surface into the fluid, rather convection of the existing vorticity downstream. Figure 21 shows the integrated convected vorticity flux for section 2 corresponding to figures $6(a, b)$ and figure 15. Also seen is a sharp increase of vorticity flux at $3.6 \mathrm{~cm}$ indicating a large injection of vorticity into the flow. The total increase is also $\sim 2250 \mathrm{~cm}^{2} \mathrm{~s}^{-2}$. Thus, the integral convective flux value is almost identical to that seen for the viscous flux integration. This clearly confirms that the vorticity is fluxed into the flow from the free surface, and is convected downstream by the shear layer, without the need to break, or entrain air into the fluid.

\subsection{Case 2: lower Froude and Reynolds numbers}

For the second case, the incoming velocity to the grid is $17 \mathrm{~cm} \mathrm{~s}^{-1}$. Owing to the pressure drop across the honeycomb/screen section, the fluid accelerates to a maximum velocity of $30 \mathrm{~cm} \mathrm{~s}^{-1}$. For this case, instead of the occurrence of breaking, 


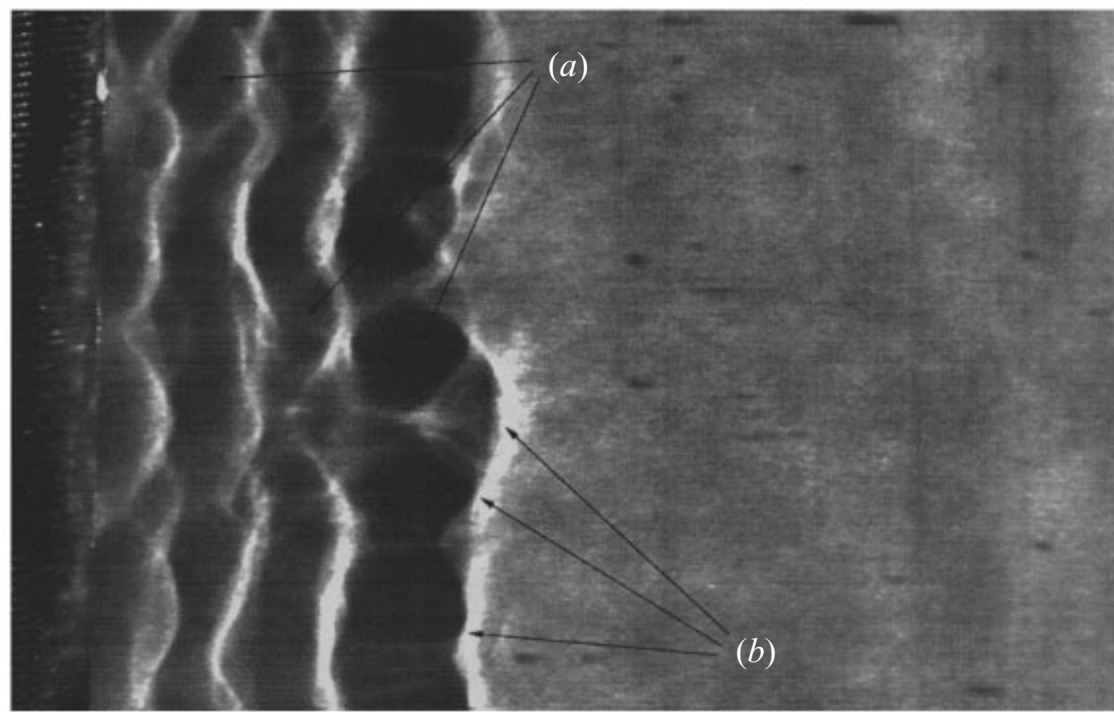

FIGURE 22. Shadowgraph for case 2. The flow is left to right: $(a)$ the capillaries; (b) the boundary at which the gravity wave begins.

a capillary-gravity wave is seen. A shadowgraph for this case is shown in figure 22, where the flow is from left to right. The capillary waves preceding wave breaking are shown by $(a)$, and the gravity wave begins and continues after $(b)$. The shadowgraph clearly shows that after the last capillary wave and into the gravity wave, the free surface does not have any small-scale deformations as in case 1, indicating there is no turbulence directly beneath the free surface. This will be further discussed below. Based on the velocity at the point immediately after the last capillary wave and the final fluid elevation after the last capillary wave, the Reynolds and Froude numbers are 1500 and 1.35 , respectively. For this case, two regions are interrogated (figure $3 b$ ). Section 1 interrogates the wave region where capillaries are seen within the imaged area, while section 2 interrogates the wave where the portion of the wave after the capillaries is seen in the imaged area. The physical dimensions of these sections are given in figure $3(b)$. The origin of these coordinates is the bottom right corner of the honeycomb/screen section (figure $2 a$ ).

\subsubsection{Velocity and vorticity fields}

The velocity and vorticity fields for this case are plotted in figures $23(a, b)$ and $24(a, b)$. The vectors within the velocity fields show the magnitude and direction of the velocity vectors throughout each of the two sections. For the vorticity plots, positive values are shown by solid lines while negative ones are shown by dashed lines. The capillaries preceding the gravity wave are $4 \mathrm{~mm}$ in wavelength, and therefore large enough for related velocity, vorticity and flux information to be extracted. The raw images further show that the amplitude (crest-to-trough height) to wavelength ratio is $\sim 0.28$. These capillaries correspond to a phase speed of $35 \mathrm{~cm} \mathrm{~s}^{-1}$ since the maximum speed of the flow prior to the gravity wave is $30 \mathrm{~cm} \mathrm{~s}^{-1}$. Therefore, the phase speed has matched the velocity just prior to wave breaking, thus exciting the capillaries. Figure $23(a, b)$ shows the velocity and positive vorticity fields at section 1 . The velocity field through the capillaries is fully attached despite the high curvature, thus resembling flow through a series of converging and diverging sections. Therefore, the turning of the flow through 

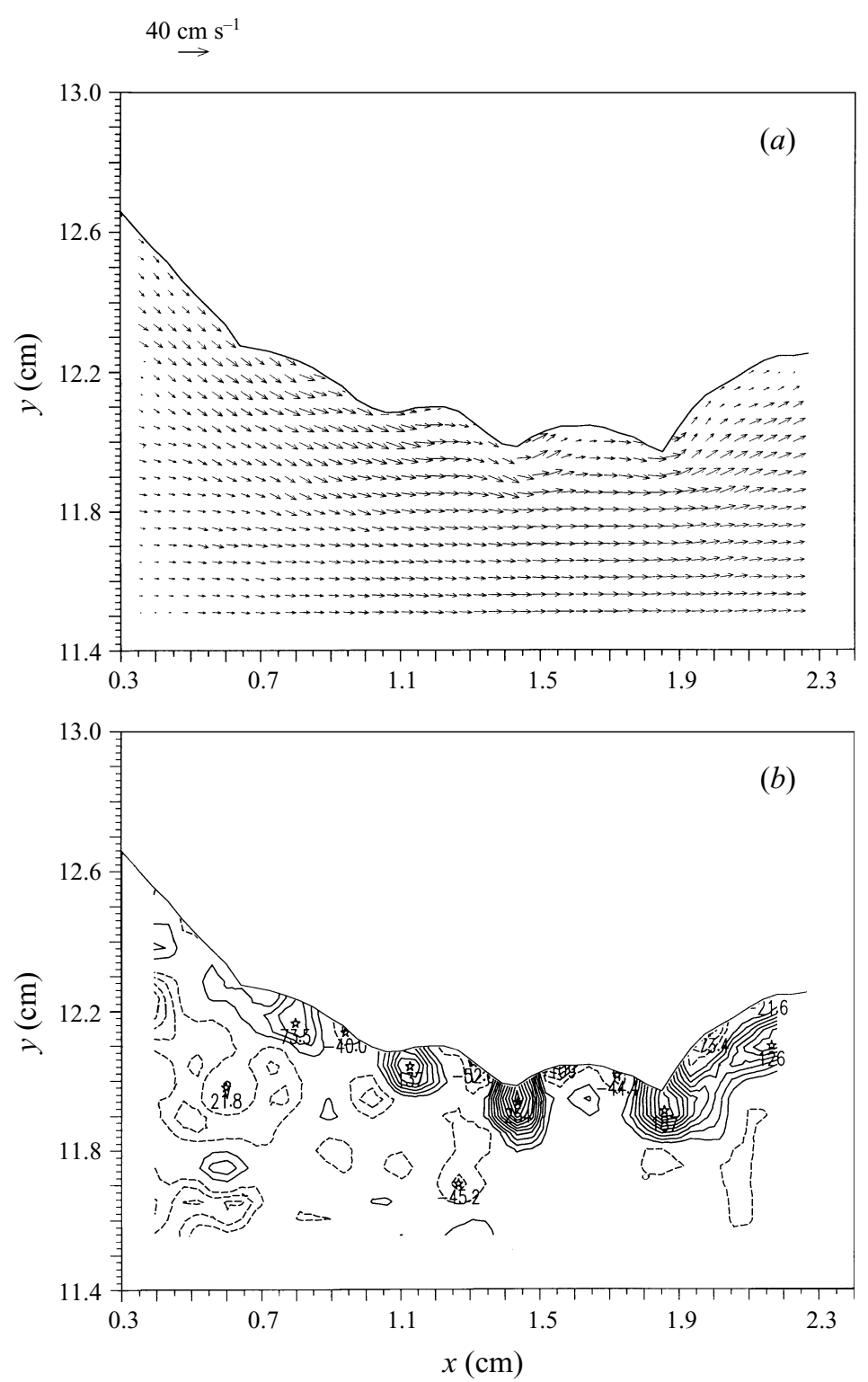

FIGURE 23. (a) Vector field of section 1 of case 2. (b) Vorticity field of section 1 of case 2. The negative contours (dashed lines) are in increments of $20 \mathrm{~s}^{-1}$ while the positive contours (solid lines) are in increments of $20 \mathrm{~s}^{-1}$.

the capillaries generates a series of quasi-stationary negative and positive regions of vorticity that exists solely due to the curvature of the free surface (Longuet-Higgins 1994). The velocity fields show that there is no separation of the flow from the free surface, or recirculation zones. Likewise, the vorticity plots show no separation of a shear layer as seen in the higher Froude number case, rather a positive shear layer is seen that bends upwards, following the contour of the gravity wave. These observations thus confirm that breaking has not occurred, as was shown in the shadowgraph for this case (figure 22). This positive vorticity layer is seen to be connected to the positive vorticity region situated under the last capillary trough just prior to the gravity wave. 

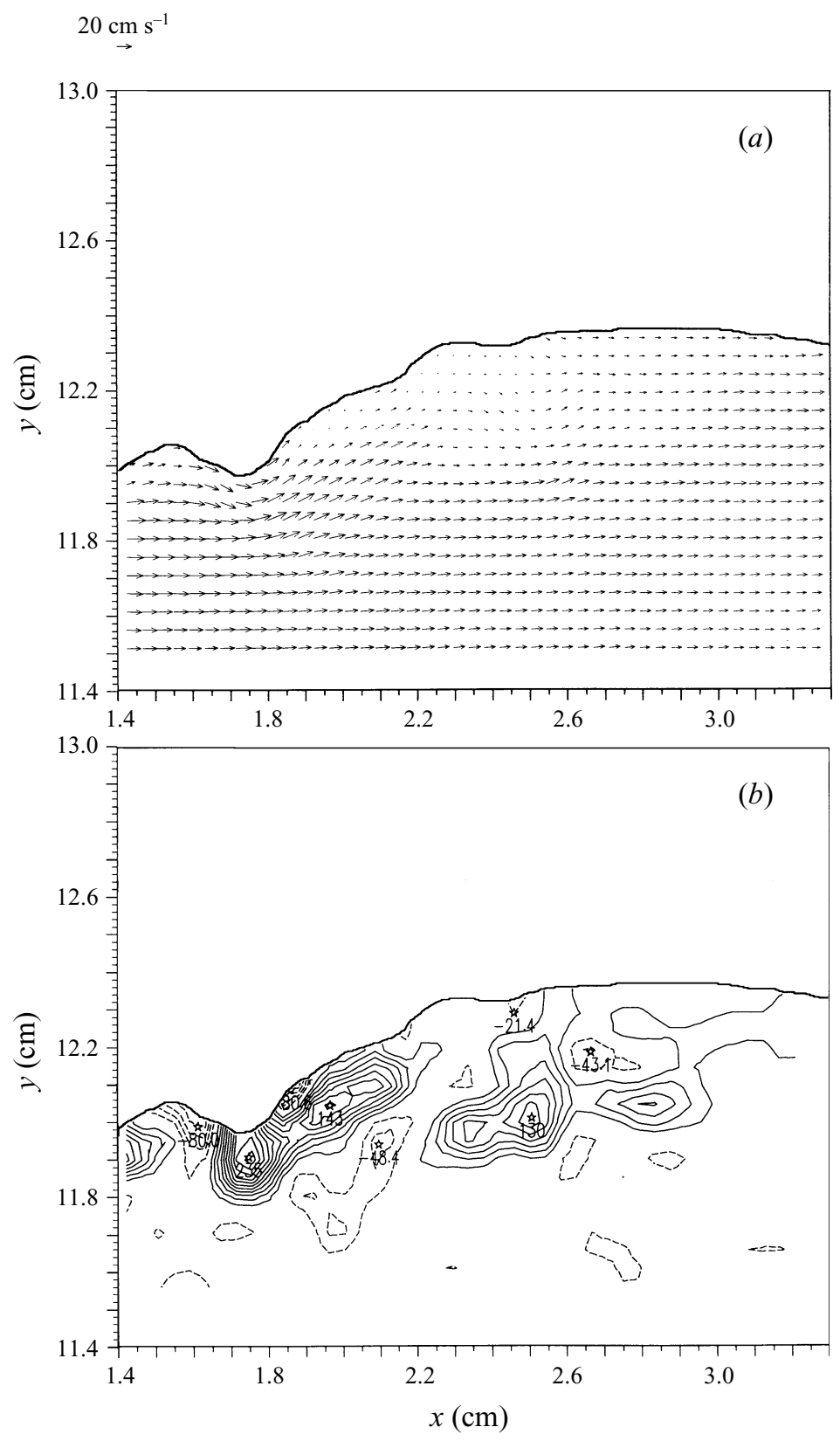

FIGURE 24. (a) Vector field of section 2 of case 2. (b) Vorticity field of section 2 of case 2. Contours as for figure $23(b)$.

Lastly, a small region of negative vorticity is seen on the gravity wave, below the surface, and above the positive shear layer at $x=1.95 \mathrm{~cm}$, indicating the existence of a stagnation point at that location.

Figure 24 $(a, b)$ shows the velocity and positive vorticity field at $\$ 2$. The vorticity field from this section confirms that the positive shear layer seen within the gravity wave does not separate from the free surface, but follows its contour. Also, it is seen that this vorticity seems to be connected to the positive vorticity located beneath the trough of 


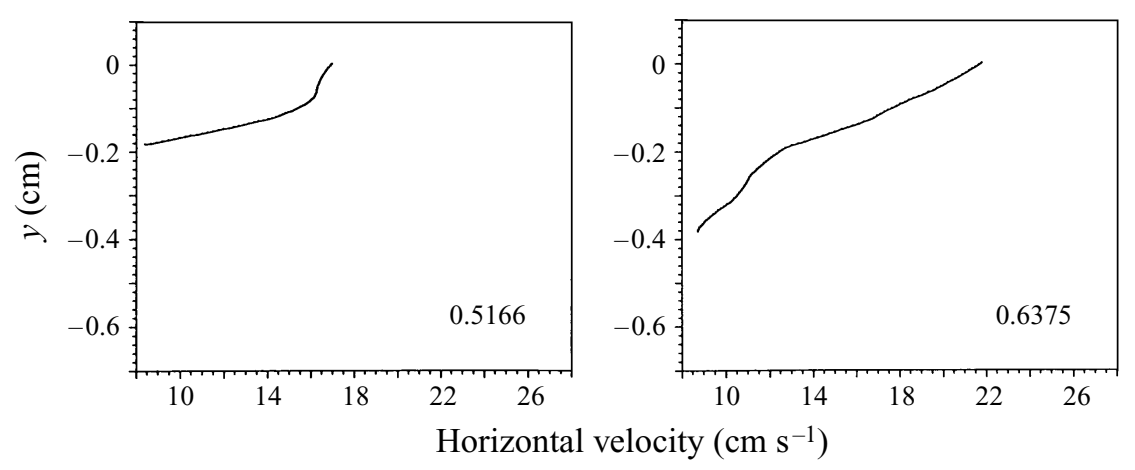

FIGURE 25. Case 2: velocity profiles perpendicular to the free surface extracted from several points through the free surface. The number on each plot indicates the $x$-axis location of the free surface through which the profile passes.

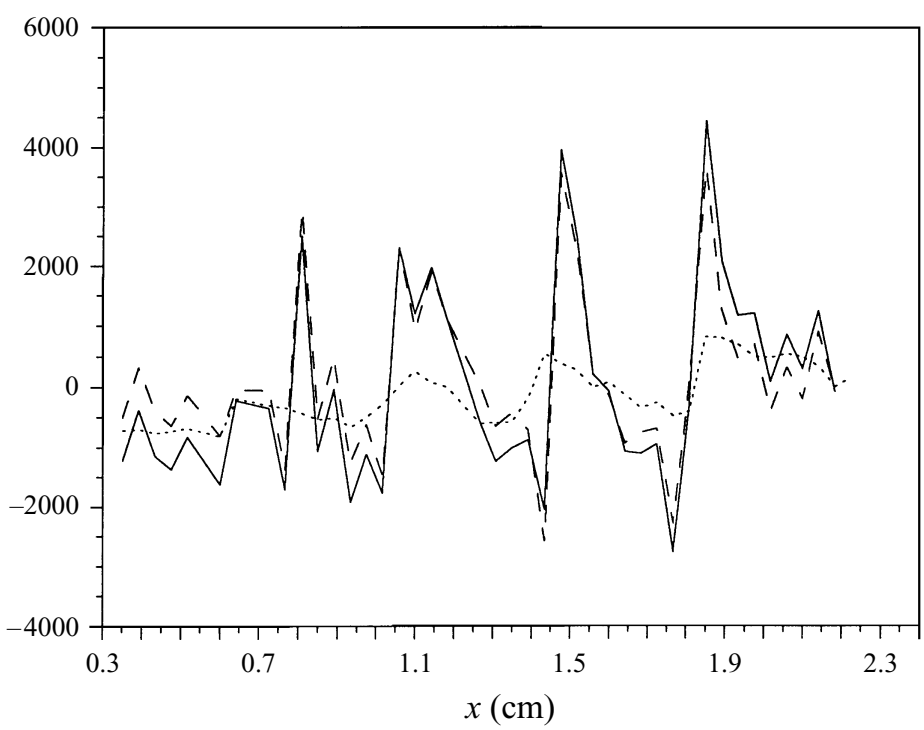

FIGURE 26. Terms contributing to the velocity flux for section 1. The kinematic deceleration is shown by the dashed line, the gravity term by the dotted line, and their sum is shown by the solid line.

that last capillary prior to wave breaking. Lastly, the small region of negative vorticity, though very small, is also present, and is located beneath the free surface and above the positive shear layer at $x=1.85 \mathrm{~cm}$.

It should be pointed out that careful observation of the velocity field shows that there is a free-surface layer, prior to the capillary waves which moves at a higher velocity than the fluid below it. Evidence of this is also seen in figure 23(b), where a relatively vorticity-free free-surface layer with a $2 \mathrm{~mm}$ thickness is seen. To verify this, velocity profiles perpendicular to the free surface were extracted at several locations prior to the capillary waves, and plotted in figure 25 . These plots show that there exists a layer of fluid that accelerates and moves at a higher velocity than the fluid beneath it. Therefore, quite clearly, there exists a free-surface layer which accelerates faster than the fluid beneath it, whereafter larger capillary waves and finally, a gravity wave are observed. This might therefore suggest that this behaviour can be described by the 


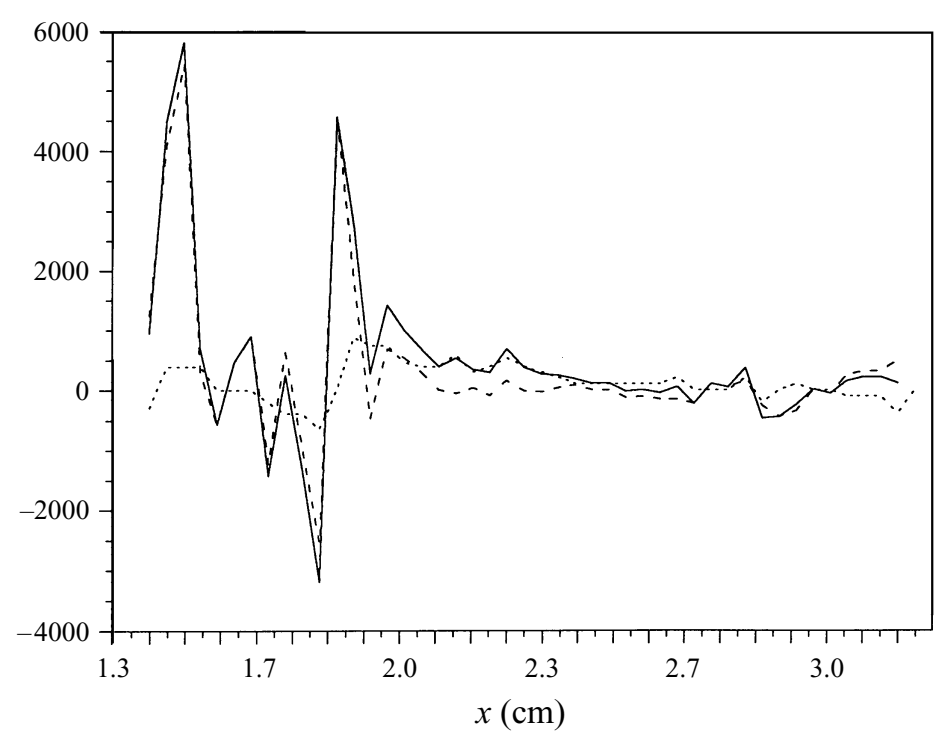

Figure 27. As figure 26 but for section 2.

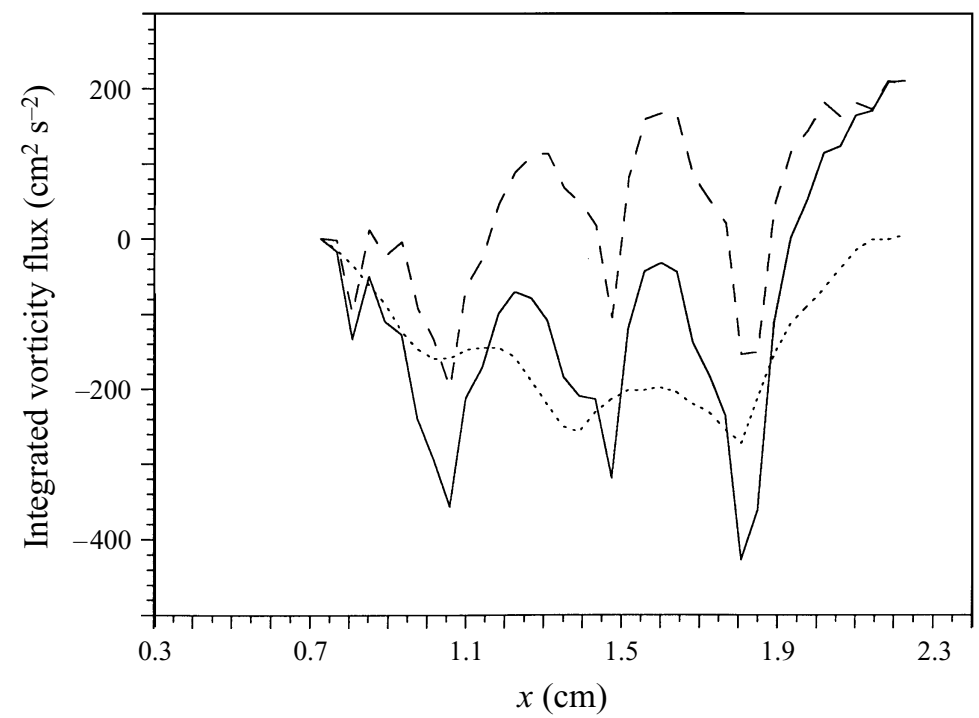

FIGURE 28. The integrated viscous vorticity flux due to the free surface deceleration (dotted line), the free surface slope (dashed line), and their sum (solid line).

hydraulic jump phenomenon described earlier. This can be checked by using the hydraulic jump relation (1). For this case, $h_{1}=0.2 \mathrm{~cm}, F r_{1}=2.14$, resulting in $h_{2}=5.14 \mathrm{~mm}$. Subtracting the pre-jump height, $h_{1}$, gives a height of $3.14 \mathrm{~mm}$, which is quite close to the observed value of $3.5 \mathrm{~mm}$ (see figure $24 a, b$ ). As in the high-speed case, the validity of the hydraulic jump assumptions must be tested. Since there is no solid lower boundary, an internal streamline must be chosen as the lower boundary. We therefore choose this streamline as our lower boundary. Assuming that the pressure in this layer is hydrostatic, we test whether the viscous force exerted on this boundary is negligible. Using this, we check condition (2). For this case, $R e_{h}=600$, 
$F r_{h}=2.14$, and the mean value of $\partial h / \partial x$ from the beginning of the gravity wave to its maximum height is 0.333 . Therefore,

$$
\left(\frac{F r_{h}^{2}}{R e_{h}} / \frac{\partial h}{\partial x}\right)=2.9 \times 10^{-2} \ll 1
$$

This condition is clearly satisfied, suggesting that this flow can be modelled as a local hydraulic jump.

\subsubsection{Experimental observations of the sources of vorticity}

Since the flow is slower in this case, the capillaries are larger, and can therefore be resolved, thus determining the importance of the gravity term in equation (8). Figure 26 shows the deceleration and gravity terms, and their sum for section 1, and how these terms vary as the fluid flows through the capillaries. It is seen that the contribution to the vorticity flux is due mostly to the deceleration term and not the gravity term. Figure 27 shows the deceleration and gravity terms, and their sum for section 2 . As for section 1 , the variations of these terms are seen as the fluid passes through the capillaries; and the contribution to the local vorticity flux is due mostly to the deceleration term and not the gravity term. Furthermore, note that the vorticity flux goes to zero after the last capillary, indicating that there is no flux of vorticity from the free surface beyond the capillaries. The integrated vorticity flux due to the free-surface acceleration, the gravity term, and their sum for section 1, corresponding to figure $23(a)$, is plotted starting from the first capillary and is shown in figure 28 . The integrated viscous vorticity flux shows that after the last trough, the final value achieved is $\sim 200 \mathrm{~cm}^{2} \mathrm{~s}^{-2}$ at $x=2.2 \mathrm{~cm}$, while that achieved by the integrated vorticity flux due to the gravity term at that point is $\sim 0 \mathrm{~cm}^{2} \mathrm{~s}^{-2}$. Thus, the capillaries do not contribute to the generation of positive vorticity. The sum of these vorticity fluxes shows that the total flux of positive vorticity is $\sim 200 \mathrm{~cm}^{2} \mathrm{~s}^{-2}$. Clearly, capillary waves are not major sources of positive vorticity generation, rather then dominant source is the deceleration of the free surface.

Aside from the visual observations that breaking does not occur for this case, the mild injection of positive vorticity, of $\sim 200 \mathrm{~cm}^{2} \mathrm{~s}^{-2}$, into the flow is further indication that breaking has not occurred, and that the phenomenon seen is a capillary-gravity wave.

\section{Conclusions}

The sources of vorticity flux are examined for a spilling wave. For the steady spilling breaker, the vorticity flux equation has contributions from two terms: the fluid deceleration term, and the gravity term. The present results reveal the underlying structure within a honeycomb/screen-generated spilling breaker responsible for the vorticity flux into the flow. This type of spilling breaker generation produces a thin higher-velocity free-surface fluid layer that decelerates just prior to breaking, thus injecting a large amount of vorticity into the fluid bulk, entirely through a viscous process. Moreover, the vorticity injection due to the free-surface deceleration is seen to dominate over the gravity-generated vorticity flux. As suggested by Banner \& Phillips, the higher Froude number data presented indicate that the breaking of the steady spilling wave is characterized by the occurrence of a stagnation point rather than by the discontinuities of the slope due to the breaker. However, further study shows that the source of the viscous vorticity flux for a steady spilling wave is due to the deceleration of the surface fluid, rather than the free-surface stagnation point or the sharp free-surface curvature at the toe. Our technique of generating a breaker allows 
sufficiently gradual deceleration along the free surface that the location of maximum deceleration, and therefore the flux of vorticity into the flow, are clearly distinguishable from the location of the stagnation point along the free surface and the onset of separation from the surface. For certain shapes of the free surface, and corresponding velocity variations along the surface, it is, in fact, possible to observe relatively large distances between these indicators. Previous investigations have not addressed these distinctions. For the type of breaker generated by Lin \& Rockwell (1994), deceleration along the free surface occurs over a distance an order of magnitude smaller than in the present study. In this respect, their breaker may be viewed as a limiting case of the ones addressed herein. The foregoing indicators leading to wave breaking are, in their case, nearly coincident. They therefore attributed the source of vorticity to be located at the sharp curvature of the free surface, where flow separation abruptly occurred.

It should be emphasized that most important is the deceleration of a free-surface layer which results in the flux of vorticity into the flow. In our case, it is shown that the higher-speed layer decelerates, resulting in an influx of vorticity. This is also seen in the hydraulic jump study of Hornung et al. (1995). Figures 14, 15 and 16 clearly show a decelerated layer at the free surface which has produced an influx of vorticity into the flow.

Case 2, the lower Reynolds and Froude numbers case, shows that the capillary curvature provides a negligible contribution of the gravity term to the vorticity flux, and that the contribution is dominated by the deceleration term as the flow passes through the capillaries. The next flux of vorticity into the flow is shown to be one order of magnitude smaller than that seen in case 1 , which would also serve as an indication of the separating shear layer in case 2. Also, the vorticity seen beneath the capillaries is due to the free-surface curvature, and while remaining at the free surface does not flux into the flow from the free surface. The vorticity is thus confined to a region within a thickness of the order of the capillary amplitude.

Finally, the structure of the spilling breaker as well as the capillary-gravity wave reveals that it might be possible to analyse spilling breakers by the shallow water hydraulic jump description, since it is the thin free-surface high-speed layer that decelerates with respect to the rest of the fluid, thus generating wave breaking in case 1 , and the capillary-gravity wave in case 2 .

This work is supported by URI research grant number N00014-92-J-1618 by the office of Naval Research. We gratefully acknowledge the helpful discussions with Dr Longuet-Higgins, Dr Doug Dommermuth, and Dr Edwin Rood that lead to our insight into this topic.

\section{REFERENCES}

Banner, M. L. \& Peregrine, D. H. 1993 Wave breaking in deep water. Ann. Rev. Fluid Mech. 25, 373-397.

Banner, M. L. \& Phillips, O. M. 1974 On the incipient breaking of small scale waves. J. Fluid Mech. 65, 647-656.

Battues, J. A. \& SaKai, T. 1981 Velocity field in a steady breaker. J. Fluid Mech. 111, 421-437.

Cointe, R. \& Tulin, M. 1994 A theory of steady breakers. J. Fluid Mech. 276, 1-20.

Cox, C. S. 1958 Measurements of slopes of high-frequency wind waves. J. Mar. Res. 16, 199-225.

DunCAN, J. H. 1981 An experimental investigation of breaking waves produced by a towed hydrofoil. Proc. R. Soc. Lond. A 377, 331-348.

Duncan, J. H. 1983 The breaking and non-breaking wave resistance of two-dimensional hydrofoil. J. Fluid Mech. 126, 507-520. 
Duncan, J. H. \& Philomin, V. 1994 The formation of spilling breaking water waves. Phys. Fluids 8, 2558-2560.

Gharib, M. \& Weigand, A. 1996 Experimental studies of vortex disconnection and connection at a free surface. J. Fluid Mech. 321, 59-86.

Hornung, H. G., Willert, C. E. \& Turner, S. 1995 The flow field downstream of a hydraulic jump. J. Fluid Mech. 287, 299-316.

Lin, J. C. \& Rockwell, D. 1994 Instantaneous structure of a breaking wave. Phys. Fluids 6, 2877-2879.

Lin, J. C. \& Rockwell, D. 1995 Evolution of a quasi-steady breaking wave. J. Fluid Mech. 302, $29-44$.

Longuet-Higgins, M. S. 1963 The generation of capillary waves by steep gravity waves. J. Fluid Mech. 16, 138-159.

Longuet-Higgins, M. S. 1992 Capillary rollers and bores. J. Fluid Mech. 240, 659-679.

Longuet-Higgins, M. S. 1994 Shear instability in spilling breakers. Proc. R. Soc. Lond. A 446, 399-409.

Miyata, H. \& Inui, T. 1984 Nonlinear ship waves. Adv. Appl. Mech. 24, 215-288.

Peregrine, D. H. \& Svendson, I. A. 1978 Spilling breakers, bores and hydraulic jumps. Proc. 16th Coastal Engng Conf: ASCE, Hamburg, Germany, pp. 540-550.

RooD, E. P. 1994 Interpreting vortex interactions with a free surface. Trans. ASME I: J. Fluids Engng 116, 91-94.

Rood, E. P. 1995 Free-surface vorticity. In Fluid Vortices (ed. S. Green), chap. 17. Kluwer (in press).

Thompson, P. A. 1972 Compressible-Fluid Dynamics. McGraw-Hill.

Tulin, M. P. \& Cointe, R. 1986 A theory of spilling breakers. Proc. 16th Symp. Naval Hydrodynamics, Berkeley, pp. 93-105. National Academy Press, Washington, DC.

Willert, C. E. \& Gharib, M. 1991 Digital particle image velocimetry. Exps. Fluids 10, 181-193. 